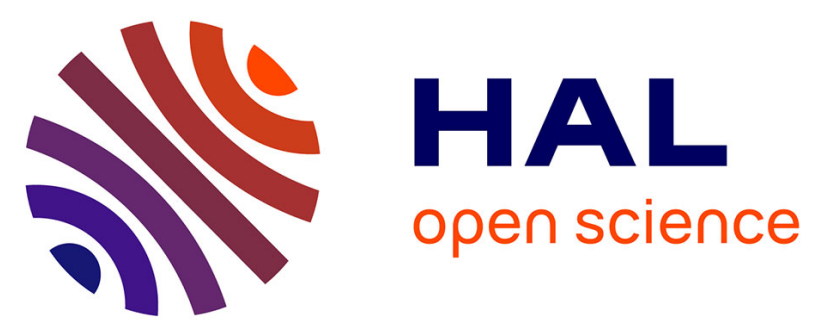

\title{
Effect of rectangular winglet pair roll angle on the heat transfer enhancement in laminar channel flow
}

Assadour Khanjian, Charbel Habchi, Serge Russeil, Daniel Bougeard, T. Lemenand

\section{To cite this version:}

Assadour Khanjian, Charbel Habchi, Serge Russeil, Daniel Bougeard, T. Lemenand. Effect of rectangular winglet pair roll angle on the heat transfer enhancement in laminar channel flow. International Journal of Thermal Sciences, 2017, 114, pp.1-14. 10.1016/j.ijthermalsci.2016.12.010 . hal-02525542

HAL Id: hal-02525542

https://univ-angers.hal.science/hal-02525542

Submitted on 31 Mar 2020

HAL is a multi-disciplinary open access archive for the deposit and dissemination of scientific research documents, whether they are published or not. The documents may come from teaching and research institutions in France or abroad, or from public or private research centers.
L'archive ouverte pluridisciplinaire HAL, est destinée au dépôt et à la diffusion de documents scientifiques de niveau recherche, publiés ou non, émanant des établissements d'enseignement et de recherche français ou étrangers, des laboratoires publics ou privés. 


\title{
Effect of rectangular winglet pair roll angle on the heat transfer enhancement in laminar channel flow
}

\author{
Assadour Khanjian a, b, c, *, Charbel Habchi ${ }^{\mathrm{d}}$, Serge Russeil a , Daniel Bougeard ${ }^{\mathrm{a}}$, \\ Thierry Lemenand ${ }^{c}$ \\ ${ }^{a}$ Mines Douai, EI, F59500 Douai, France \\ b Lebanese International University LIU, Mechanical Engineering Dept., Beirut, Lebanon \\ c Angers University, ISTIA, LARIS EA 7315, Angers, France \\ ${ }^{\mathrm{d}}$ Notre Dame University - Louaize, Mechanical Engineering Department, P.O. Box: 72 Zouk Mikael, Zouk Mosbeh, Lebanon
}

\section{A R T I C L E I N F O}

\section{Article history:}

Received 27 May 2015

Received in revised form

20 October 2016

Accepted 13 December 2016

\section{Keywords:}

Longitudinal vortices

Heat transfer enhancement

Rectangular winglet

Nusselt number

Friction factor

Energy efficiency

\begin{abstract}
A B S T R A C T
Convective heat transfer enhancement can be achieved by generating secondary flow structures that are added to the main flow to intensify the fluid exchange between hot and cold regions in the system. One method involves the use of vortex generators to produce streamwise and transverse vortices on the top of the main flow. This study presents numerical computation results on laminar convection heat transfer in a rectangular channel which bottom wall is equipped with rectangular winglet pair vortex generators. The governing equations are solved using finite volume method by considering steady state, laminar regime and incompressible fluid. Three dimensional numerical simulations are performed to study the effect of the generators' roll-angle $\beta$ on the flow and heat transfer characteristics. Different values of rollangle $\beta$ in the range $\left[20^{\circ}-90^{\circ}\right]$ are considered, while maintaining a constant angle of attack $\left(\alpha=30^{\circ}\right)$ for all the cases. The influence of the Reynolds number is also studied for values 456 and 911 (based on the channel hydraulic diameter). Both local and global analyses of the thermal performances are carried out using parameters such as the Nusselt number and the friction coefficient. In addition, the position and strength of the longitudinal vortices created are presented and discussed, highlighting their effect on the heat transfer rates throughout the duct, for the various generators' roll-angles. Finally, from both local and global investigations, it is found that the optimal values of the roll-angle, determined for each Reynolds number, are not necessarily $90^{\circ}$, which corresponds to the widely used configuration in the open literature.
\end{abstract}

(c) 2016 Published by Elsevier Masson SAS.

\section{Introduction}

Heat transfer enhancement is essential for many engineering applications such as heat exchangers [1,2], electronic equipment [3], high temperature gas turbines [4] or nuclear power plants [5]. Among all methods existing for heat transfer enhancement, some are based on the idea of generating a secondary flow, which is added to the main flow to intensify the fluid exchange between hot and cold regions in the system. One of these methods involves the use of vortex generators (VG). The VG are whether fixed on the wall or punched on it $[6,7]$.

The concept of VG was first introduced in order to improve heat

\footnotetext{
* Corresponding author. Mines Douai, EI, F59500 Douai, France.

E-mail address: assadour.khanjian@liu.edu.lb (A. Khanjian).
}

transfer with a relatively minor increase in pressure losses [6,8-10]. There are different techniques of usage of VG. Based on their function, these techniques can be divided into three categories: active VG, passive VG, and compound VG [11]. Active VG need external power like electric or acoustic fields, mechanical devices or surface vibration. On the other hand, passive VG do not need external power but use special aerodynamic surface geometry to create longitudinal or roll vortices. Four basic configurations of vortex generators (VG) are widely discussed in the open literature: delta wings (DW), rectangular wings (RW), delta-winglet pair (DWP), and rectangular winglet pair (RWP). When the trailing edge is attached to the plate, the VG is a wing, when the chord length is attached to the plate, it is called a winglet [6]. Other shapes of VG may also be found in recent papers like trapezoidal VG in the high efficiency vortex (HEV) static mixer [12,13]. There are other geometries for the VG, Zhou et al. [14] studied the heat transfer 
enhancement due to linear and curved VG having a punched holes on them, whereas Wang et al. [15] investigated the semi dimple VG.

There are two major types of vortices generated by VG: transverse vortices (TV) and longitudinal vortices (LV). Transverse vortices are mainly recirculation regions in the wake of the VG and affect unfavorably the heat transfer locally in that region. These vortices may induce local overheated regions which may be critical in case of exothermal chemical reactions for example [13]. Meanwhile, longitudinal vortices positively act upon heat transfer through three mechanisms: developing boundary layers, vortices and flow destabilization. New boundary layers will be generated around the surface of the VG, vortices will be generated as a result of the VG and flow will be less stable due to the reversed flows created because of the VG. LV are 3D swirling motion and persist over a long distance downstream the VG in the flow direction.

Several experimental and numerical investigations were conducted to study the heat transfer enhancement downstream from VG. Wu et al. [16] studied the effect of the location of the LVG along the channel and its dimensions and shape on the heat transfer enhancement and pressure loss in a rectangular channel for Reynolds number of $R e=1600$. Based on their results, they concluded that increasing the area of the VG will result in increasing both heat transfer and pressure loss. Also, by making some changes in the dimensions of the VG, like increasing the span and decreasing the height, in a way that the area of the VG is kept constant, it will lead to achieve higher heat transfer enhancement while maintaining, or even decreasing, the pressure loss in the channel. Due to the presence of the VG the cross-sectional flow area of the channel decreases, and the fluid velocity around the VG increases for the same flow rate. As a result, the strength of the generated LV increases leading to an enhancement in the heat transfer. On the other hand, this decrease in the cross-sectional flow area of the channel will lead to a pressure drop.

Wu et al. [17] studied numerically the laminar convection in a channel with punched rectangular winglet pairs. They found that heat transfer enhancement increases with the increase of Reynolds number and angle of attack and the pressure drop increases rapidly with the increase of the angle of attack.

Tiggelbeck et al. [18] investigate experimentally the heat transfer enhancement and pressure losses for different configurations of VG (DW, RW, DWP and RWP) in developing turbulent flow. Based on their results, they concluded that in a rectangular channel, the winglet VG gives better performance than the wings.

In the open literature, most of the studies focus on the effect of the VG aspect ratio, its attack angle and flow rate on the heat transfer enhancement and pressure losses. To our knowledge, there is no study for the effect of the roll-angle $\beta$ on the heat transfer process. Thus, in the present study, numerical investigation is performed on the effect of the roll-angle of RWP on the thermal performance in laminar viscous flow between two parallel plates.

Different values of roll-angle $\beta$ in the range $\left[20^{\circ}-90^{\circ}\right]$ are considered, while maintaining constant angle of attack $\left(\alpha=30^{\circ}\right)$ strikethrough. This value for $\alpha$ is proposed based on the results obtained by the experimental study of the Tiggelbeck et al. [18].

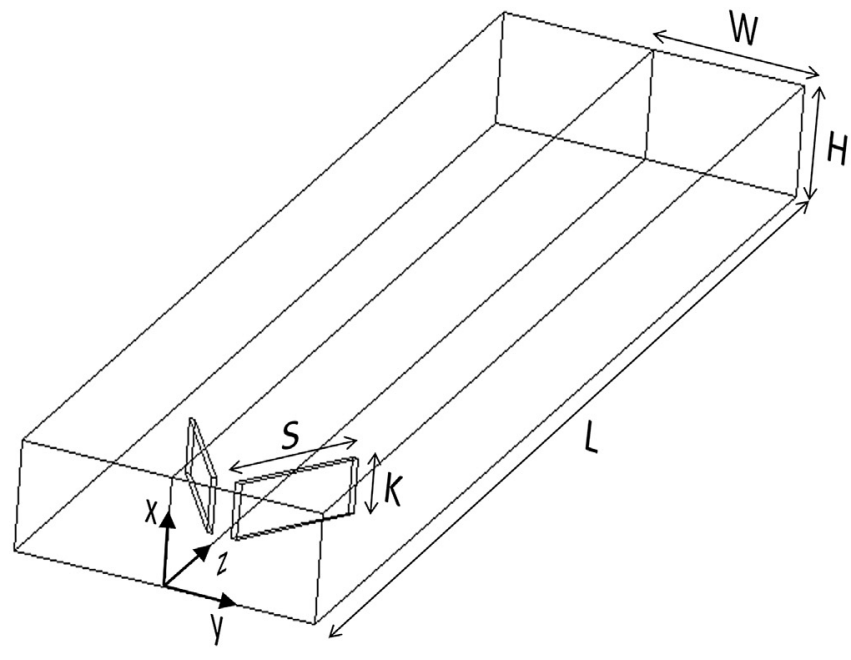

(a)
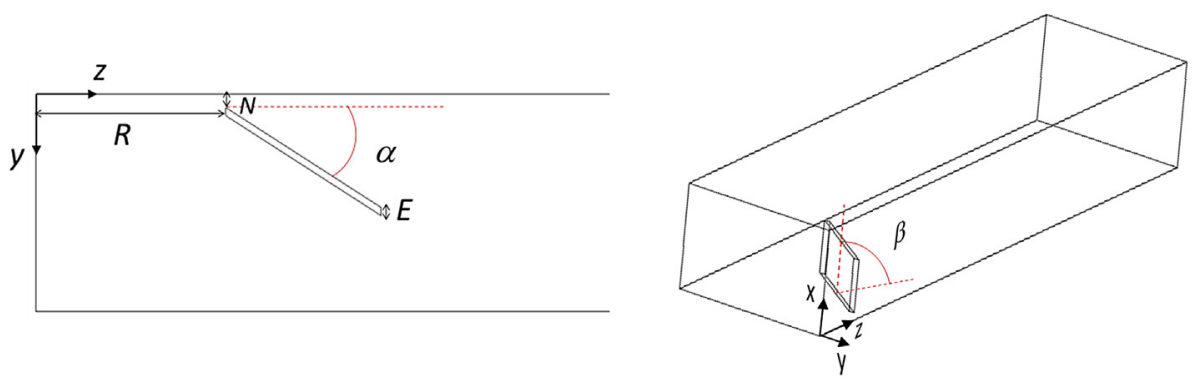

(b)

(c)

Fig. 1. (a) Isometric view of the computational domain, (b) top view showing the attack angle $\alpha$ and (c) the isometric view where angle $\beta$ is represented. 
Local and global parameters of the Nusselt number and the friction coefficient are studied. Also, the position and strength of the vortices being created in the duct are studied highlighting their effect on the heat transfer performance.

\section{Study case description}

\subsection{Governing equations}

The flow field is governed by three-dimensional (3D) steadystate continuity and momentum equations. The fluid is considered to be incompressible Newtonian with constant properties. The flow is considered as to laminar flow and the governing equations can be expressed as follows:

Continuity equation : $\nabla \cdot \vec{u}=0$

Momentum equation : $\rho(\vec{u} \cdot \nabla) \vec{u}=-\nabla p+\mu \nabla^{2} \vec{u}$

Energy equation : $\rho C_{p} \nabla \cdot(\vec{u} T)=k_{f} \nabla \cdot(\nabla T)$

where $\vec{u}$ is the velocity vector, $C_{p}$ is the specific heat at constant pressure, $p$ the pressure, $\mu$ the fluid viscosity, $T$ the temperature, $\rho$ is the density of the fluid and $k_{f}$ the fluid thermal conductivity.

Table 1

Dimensions of the computational domain and VG.

\begin{tabular}{lll}
\hline Channel height & $\mathrm{H}$ & $38.6 \mathrm{~mm}$ \\
Channel width & $\mathrm{W}$ & $1.6 \mathrm{H}$ \\
Channel length & $\mathrm{L}$ & $13 \mathrm{H}$ \\
Angle of attack & $\alpha$ & $30^{\circ}$ \\
Roll angle & $\beta$ & $20^{\circ}-90^{\circ}$ \\
VG length & $\mathrm{S}$ & $1.5 \mathrm{H}$ \\
VG height & $\mathrm{K}$ & $0.5 \mathrm{H}$ \\
Distance from the inlet to VG & $\mathrm{R}$ & $1.55 \mathrm{H}$ \\
VG thickness & $\mathrm{E}$ & $0.052 \mathrm{H}$ \\
Distance from the symmetry wall to VG & $\mathrm{N}$ & $0.1 \mathrm{H}$ \\
\hline
\end{tabular}

\subsection{Numerical procedure}

The computational fluid dynamics (CFD) software used to compute the Navier-Stokes and energy equations is STAR-CCM+ [19], which is based on the finite volume method. The segregated flow solver is used where the equations are computed in a segregated manner, i.e. one for each component of velocity, and one for pressure. The coupling between the momentum and continuity equations is achieved with a predictor-corrector approach. The pressure-velocity coupling is performed with the SIMPLE algorithm. The convective terms in the governing equations for momentum and energy are discretized with the second-order upwind scheme and second-order central scheme for diffusion terms. The algebraic multi-grid (AMG) linear solver is used to solve the velocity, pressure and temperature with Gauss-Seidel relaxation scheme.

The residual value $10^{-14}$ [19] is considered as the convergence criterion for the solutions of the flow and the energy equations. Beyond this value, the equations are solved with no significant changes in the values for velocity and temperature.

\subsection{Geometry, computational domain and boundary conditions}

The whole geometry of our study is consisted of one row of RWP placed in between two parallel plates as seen in Fig. 1(a). The computational domain consists of a rectangular duct of height $H=38.6 \mathrm{~mm}$, width $W=1.6 H$ and a length $L=13 H$ in which a RWP is inserted near the entrance on the bottom wall at a distance $R=1.55 \mathrm{H}$ away from the inlet as shown in Fig. 1 . The origin of the coordinate system is located on the computational domain as shown in Fig. 1(c). As stated in the introduction, the attack angle $\alpha$, which is the angle between the VG reference line and the incoming flow in the $Z$ direction, is fixed to $30^{\circ}$ (see Fig. 1(b)) based on the experimental results [18], while the roll-angle $\beta$ which is the angle between the plane of the VG measured with respect to the bottom wall and it varies between $20^{\circ}$ and $90^{\circ}$ (see Fig. 1(c)). The rest of the dimensions are given in Table 1 in term of the channel height $H$.

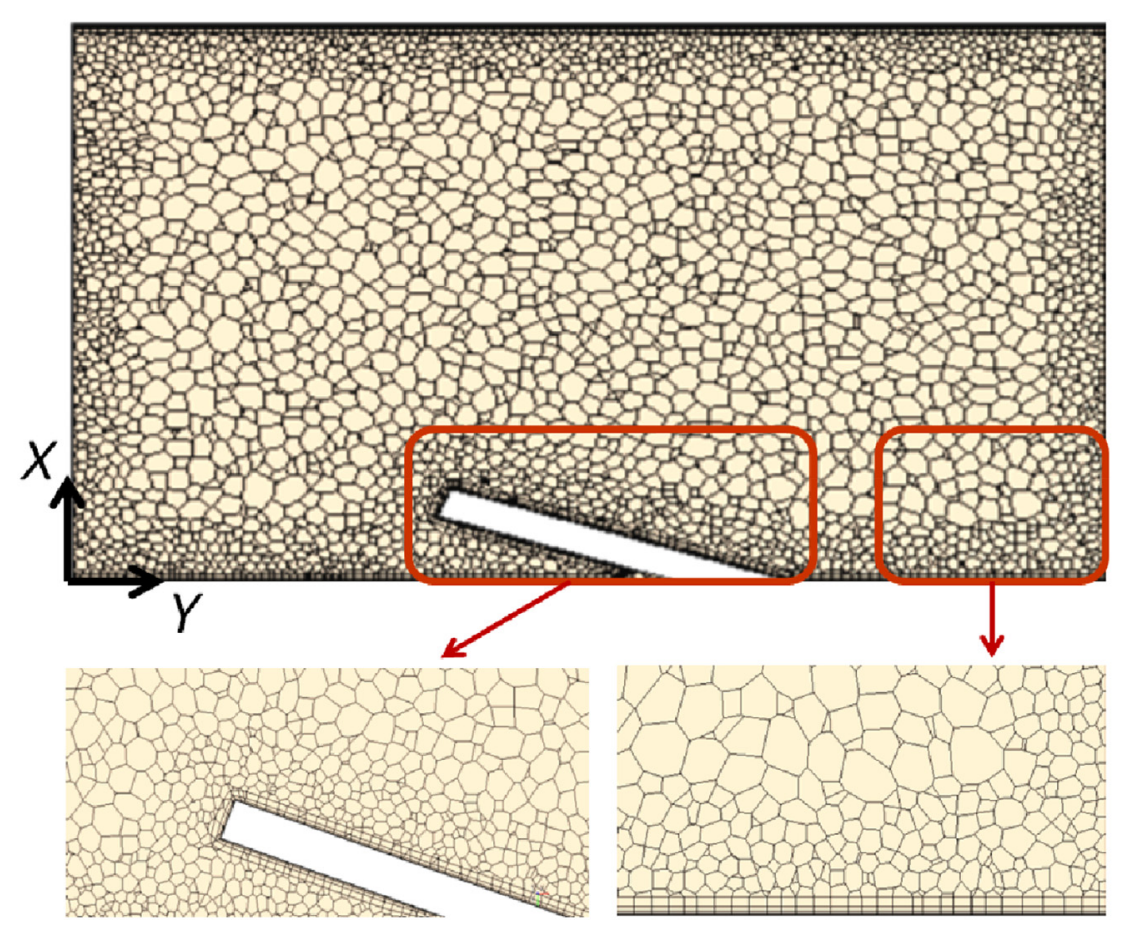

Fig. 2. Example of the mesh on a cross section showing the refinements around the VG and near the walls for $\beta=20^{\circ}$. 
Taking into account that both sides are considered to be symmetry relative to the plan $(X, Z)$, only half of the domain is computed. Flow and heat transfer simulations are carried out for Reynolds numbers 456 and 911, with uniform inlet temperature set to $T_{\text {in }}=293 \mathrm{~K}$ and wall surface temperature $T_{w}=333 \mathrm{~K}$ set for all the both bottom and top walls. Also the temperature of the VG is set to be at $T_{w}=333 \mathrm{~K}$.

\subsection{Mesh study}

A non-uniform polyhedral mesh is generated in the core of the computational domain. Near the wall and VG surfaces, a prismlayer refinement mesh is preferred due to the presence of high velocity, temperature and pressure gradients in these regions. An example of the mesh on a flow cross section is shown in Fig. 2.

To determine the appropriate mesh density, the solver is run with increasing mesh densities until no significant effect on the results is detected. Where the solution is reached to a grid independent solution where the results change so little with a denser or looser grid where the errors can be neglected in numerical simulation. The mesh validity verification is performed first by using the method proposed by Celik et al. [20] where the grid convergence index (GCI) and the apparent order of convergence (c) can be obtained. In the present study the mesh refinement is assessed by means of the global Nusselt number which is a major interest in our study. This dimensionless number represents the ratio of convective to conductive transfer defined as:

$N u=\frac{h D_{h}}{k_{f}}$

where $h$ is the heat transfer coefficient $\left(\mathrm{W} / \mathrm{m}^{2} . \mathrm{K}\right), D_{h}$ is the hydraulic diameter equal to $2 H(\mathrm{~m})$ and $k_{f}$ is the thermal conductivity of the working fluid (air) (W/m.K).

In this equation, the global heat transfer coefficient is obtained from the logarithmic mean temperature difference:

$h=\frac{\dot{q}}{A \Delta T_{l m}}$

where $\dot{q}$ is the global rate of heat transfer $(\mathrm{W})$ defined in equation (6), $A$ is the heat transfer surface area $\left(\mathrm{m}^{2}\right)$ and $\Delta T_{l m}$ is the logarithmic mean temperature difference defined in equation (7),

$\dot{q}=\dot{m} C_{p} \Delta T$

where $\dot{m}$ is the mass flow rate ( $\mathrm{kg} / \mathrm{s}), C_{p}$ is the specific heat (J/kg.K) and $\Delta T$ is the temperature difference between the flow inlet and outlet $\left(\Delta T=\left(T_{\text {out }}-T_{\text {in }}\right)\right)$ (in $\left.\mathrm{K}\right)$.

$\Delta T_{l m}=\frac{\left(T_{\text {in }}-T_{w}\right)-\left(T_{\text {out }}-T_{w}\right)}{\ln \left(\frac{T_{\text {out }}-T_{w}}{T_{\text {in }}-T_{w}}\right)}$

where $T_{\text {in }}$ is the inlet bulk temperature (K), $T_{\text {out }}$ is the outlet bulk temperature and $T_{w}$ is the wall temperature (K).

The different mesh densities and their features are represented

Table 2

Mesh characteristics.

\begin{tabular}{llll}
\hline Mesh & M0 & M1 & M2 \\
\hline Number of cells & 621,424 & $1,251,620$ & $2,711,600$ \\
Mesh size & 0.369 & 0.293 & 0.226 \\
Refinement factor & - & 1.3 & 1.3 \\
\hline
\end{tabular}

in Table 2. The mesh size is obtained by a relation of the cell volume and the total number of cells as mentioned in Table 2. It is desirable that the grid refinement factor be greater than 1.3 as proposed by Celik et al. [20]. Based on equations (4)-(7), the Nusselt number is calculated for each mesh density then the convergence order $c$ and

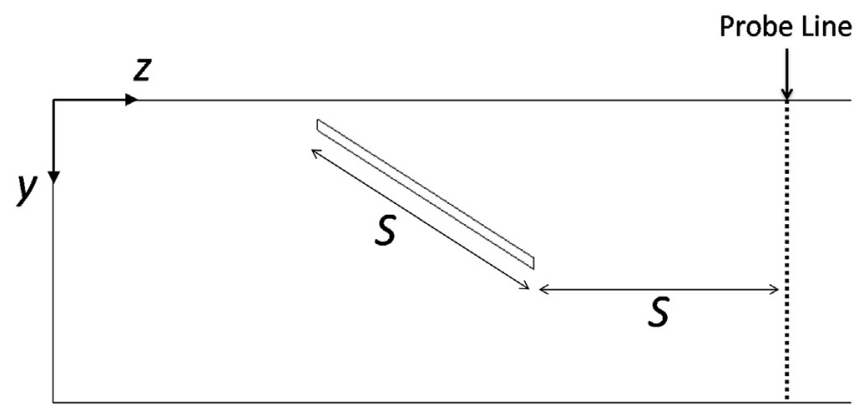

Fig. 3. Top view of the computational domain showing the probe line created downstream the VG for local mesh analysis.

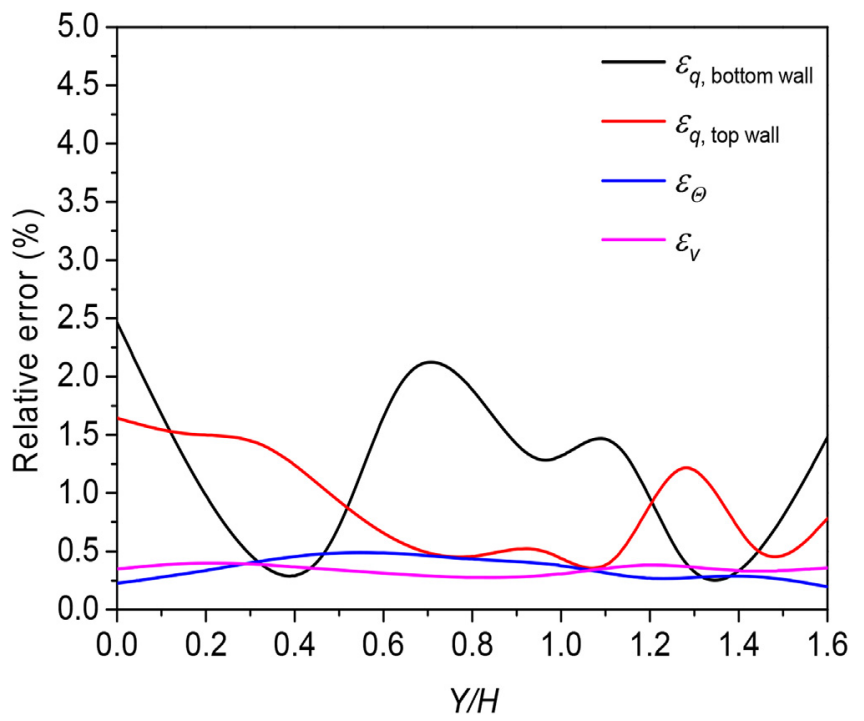

Fig. 4. Relative local error for bottom and top wall heat fluxes, velocity and dimensionless temperature between the meshes M1 and M2.

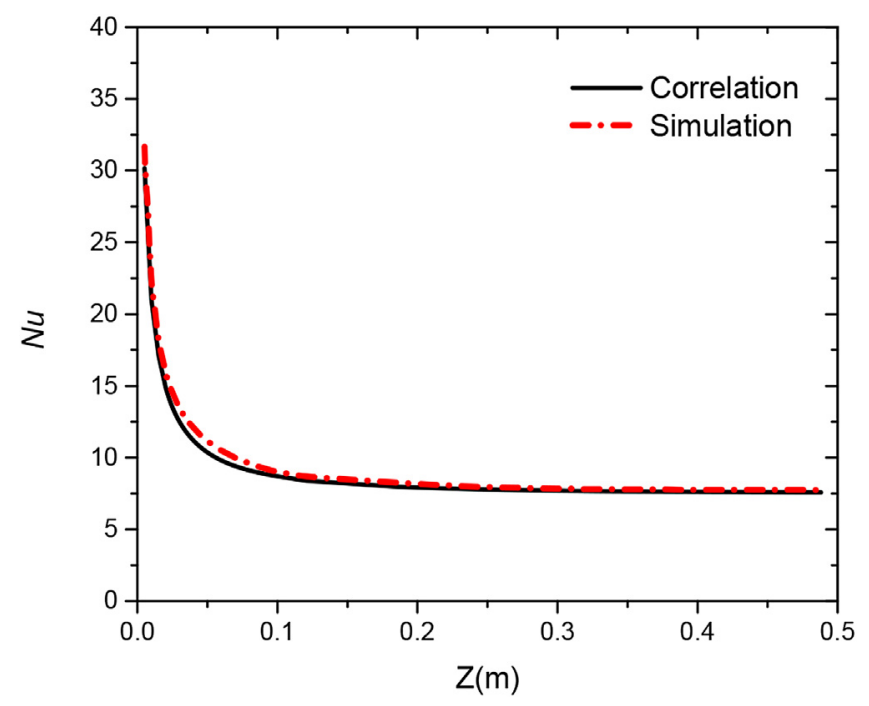

Fig. 5. Local Nusselt number validation for the empty channel case. 
GCI are computed based on the method proposed by Celik et al. [20].

Hence, it is found that the uncertainty in the fine-grid solution is GCI $1.25 \%$ and the convergence order is $c=5.97$, which are both accepted values for which the results are considered to be grid independent. For more details about the calculation of $c$ and GCI the reader can refer to Celik et al. [20]. It should be noted that the mesh study presented here is done on the highest Reynolds number
$(\operatorname{Re}=911)$.

Additionally local mesh study is also performed by considering a transversal probe line located at a distance $S$ downstream from the winglet (see Fig. 3) on both bottom and the top walls of the duct, where the wall heat fluxes transversal profiles can be analyzed. Another probe line is created in the core flow, at the center of the duct $(H / 2)$ and at the same distance downstream from the winglet, to enable the velocity and temperature transversal profiles analysis

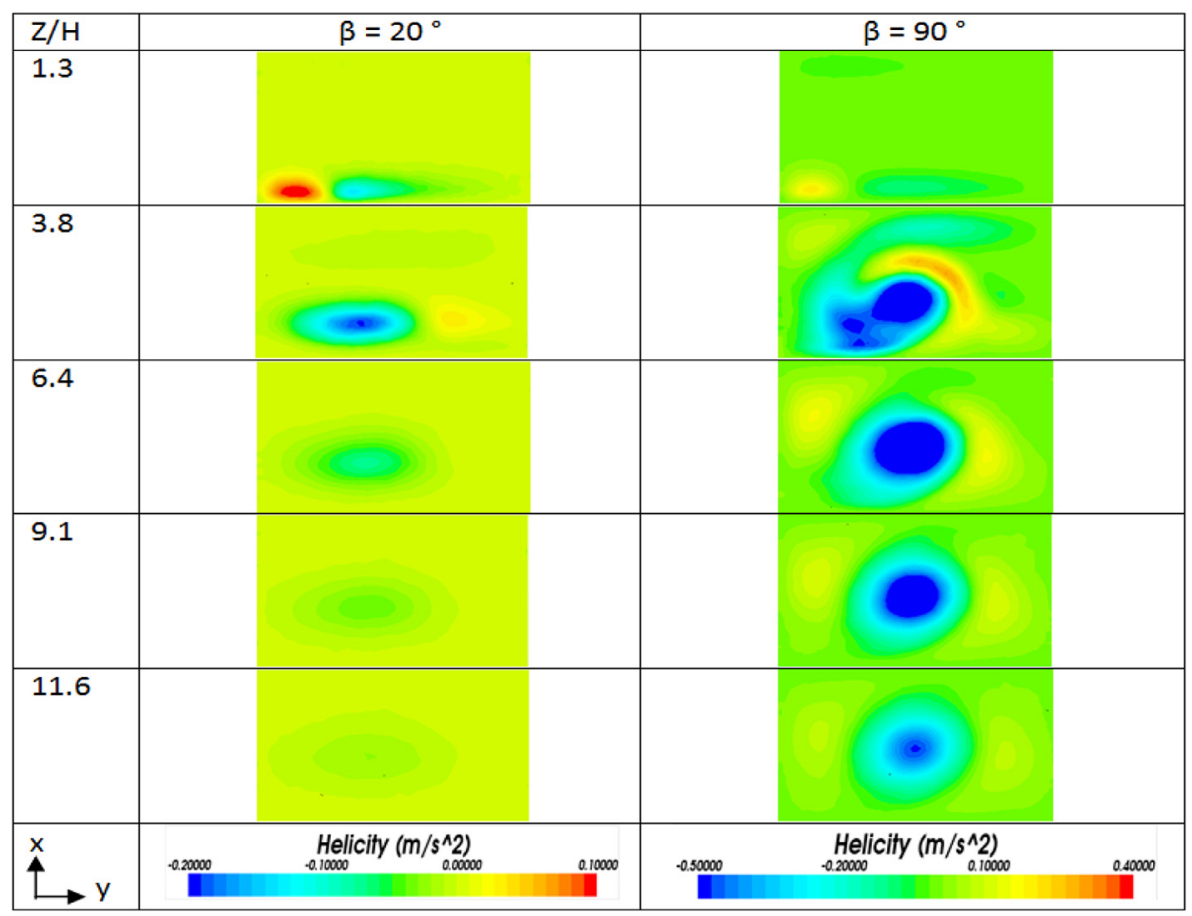

(a)

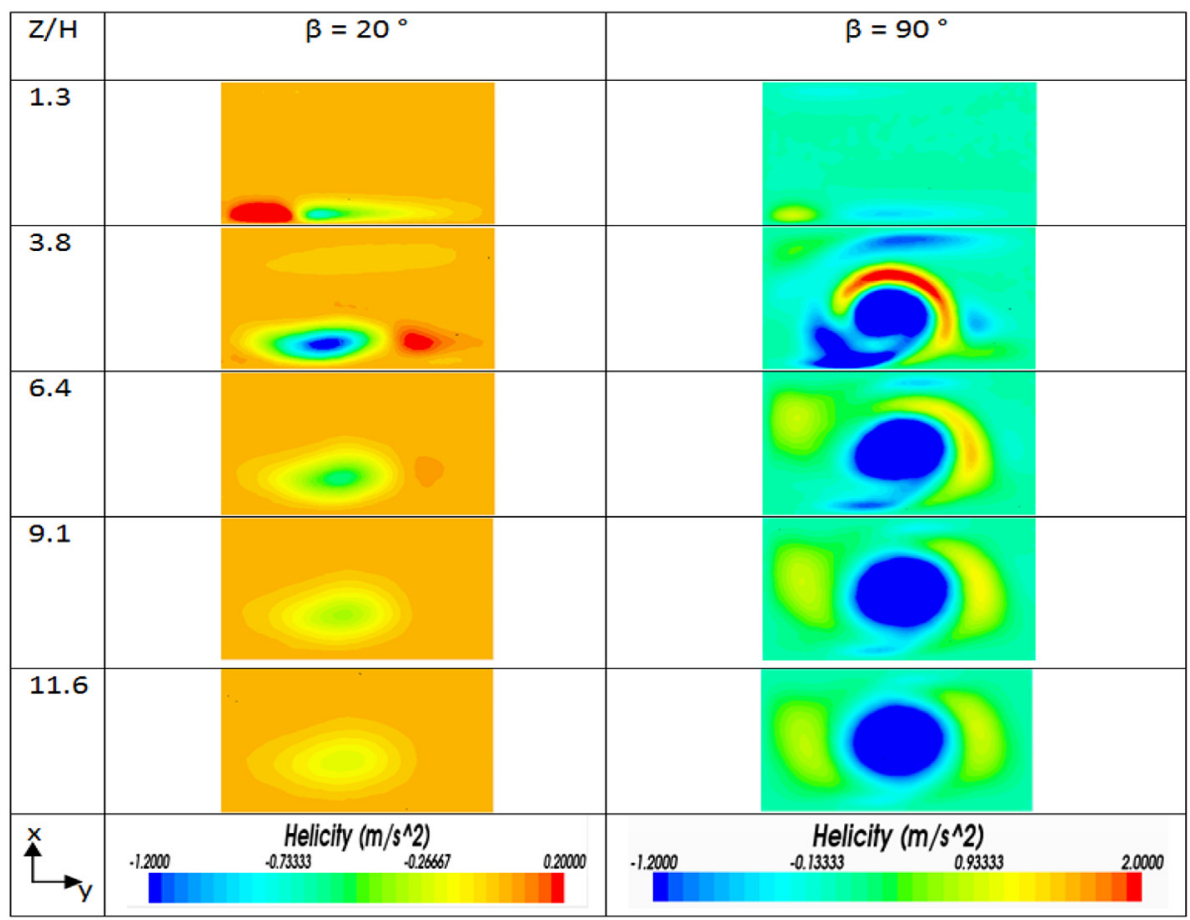

(b)

Fig. 6. Helicity distribution for (a) $R e=456$ and (b) $R e=911$ on different flow cross sections. 
for each mesh density considered.

The local relative errors between mesh densities M1 and M2 are calculated for various variables using the following equations:

- for heat flux on the top and bottom walls:

$\varepsilon_{q}=\left|\frac{q_{M 1}-q_{M 2}}{q_{M 2}}\right|$

- for velocity in the core flow $(H / 2)$ :

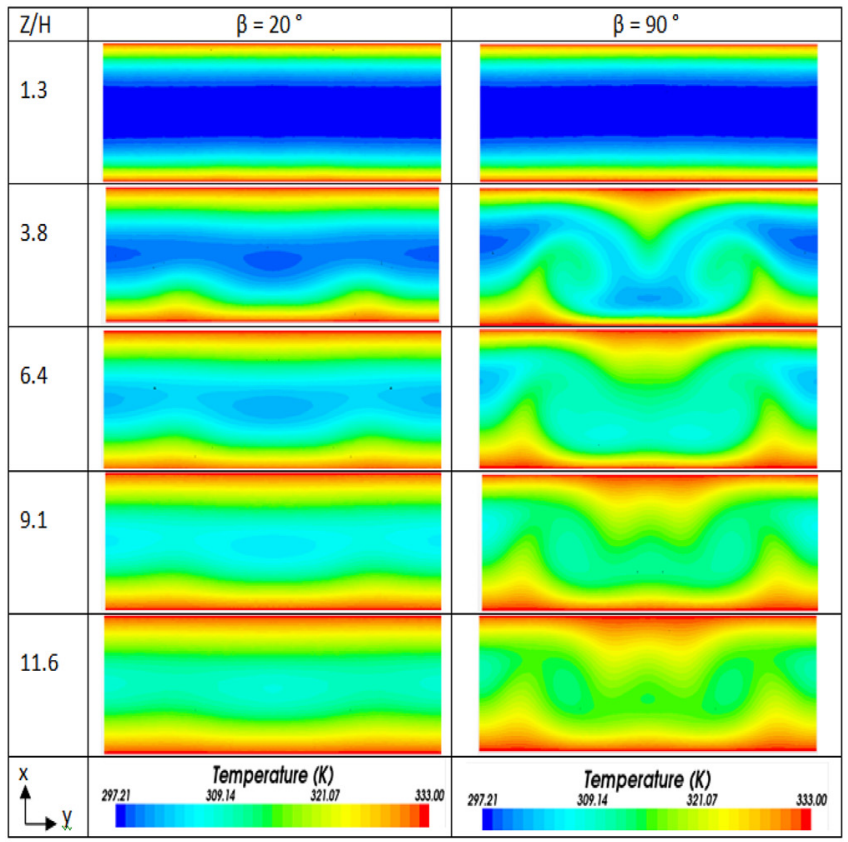

(a)

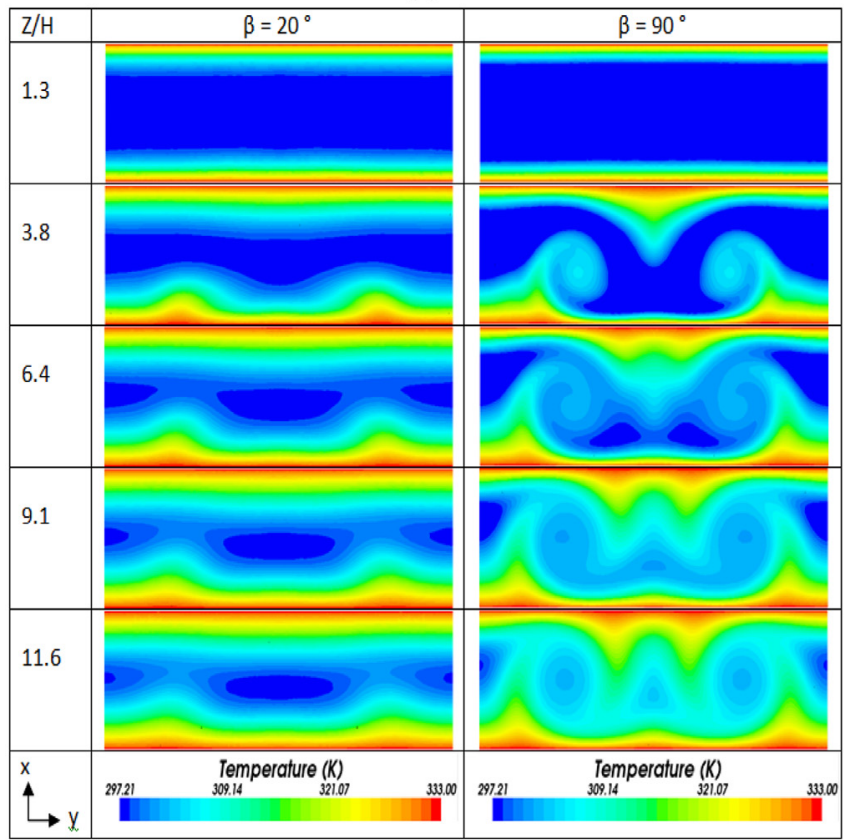

(b)

Fig. 7. Temperature distribution for (a) $R e=456$ and (b) $R e=911$ on different flow cross sections.
$\varepsilon_{v}=\left|\frac{v_{M 1}-v_{M 2}}{v_{M 2}}\right|$

- for dimensionless temperature $\Theta$ at mid-channel height:

$\Theta=\frac{T-T_{w}}{T_{i n}-T_{w}}$

$\varepsilon_{\Theta}=\left|\frac{\Theta_{M 1}-\Theta_{M 2}}{\Theta_{M 2}}\right|$

Based on the results presented in Fig. 4, it can be seen that the relative errors between the M1 and M2 mesh densities always do not exceed $2.5 \%$.

To verify the unsteadiness of the flow, numerical simulations where run using the unsteady laminar solver. After observing the

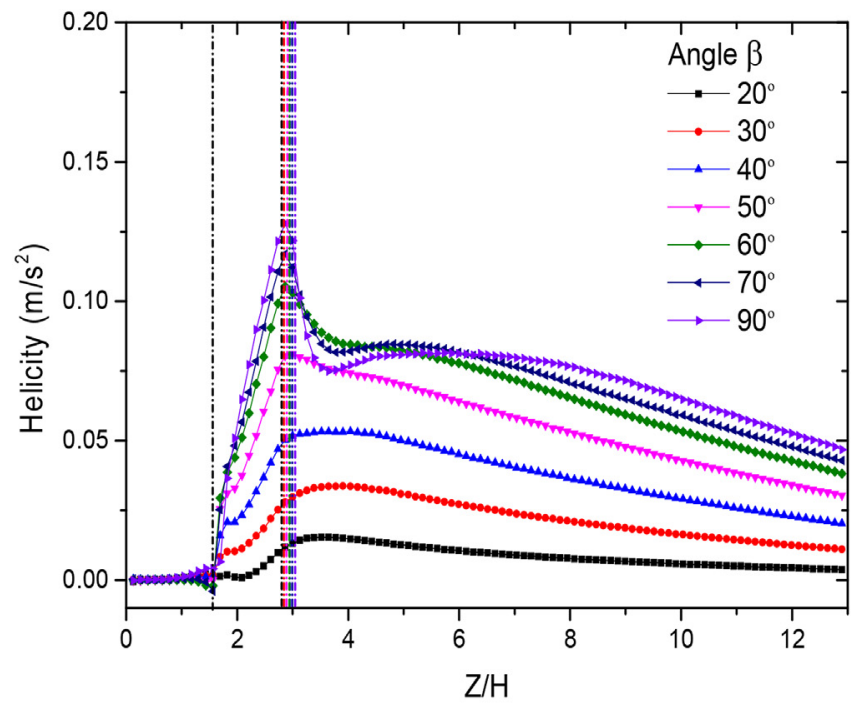

(a)

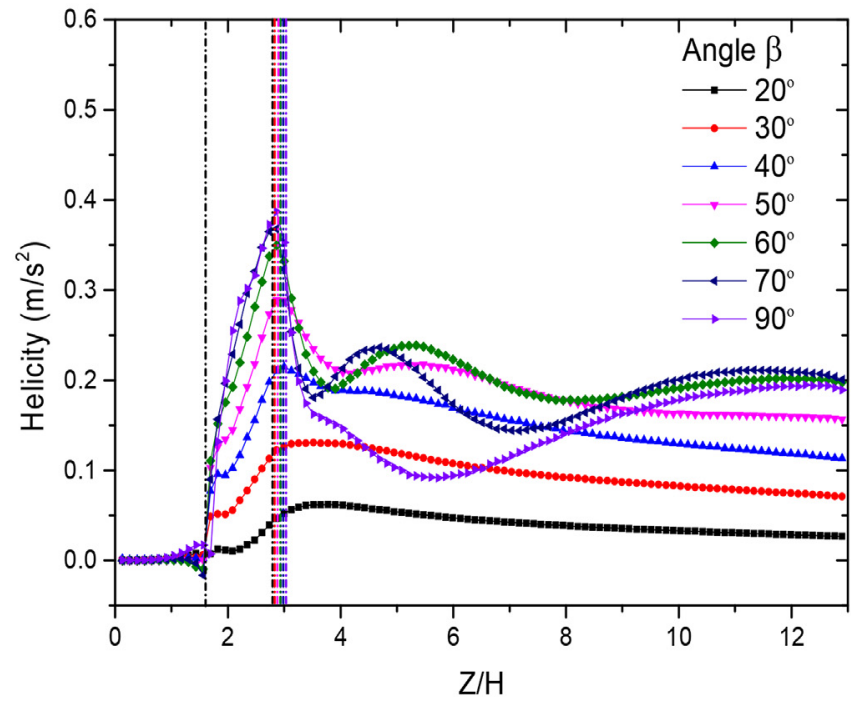

(b)

Fig. 8. Variation of averaged helicity along the duct for (a) $R e=456$ and $R e=911$. 
temporal variation of the velocity and temperature fields, it was concluded that there is no unsteadiness. The time variation of the rate of heat transfer was of the order of $10^{-4} \mathrm{~W}$ which is negligible. The steady laminar flow assumption was also made in several studies from the open literature such as Lu and Zhou [21] and Dezan et al. [22].

Moreover, additional numerical simulations were run for the empty channel computational domain for both Reynolds numbers and for two different cases: in the first the density is kept constant and there are no gravity effects, whereas in the second case we use the Boussinesq approximation for the air properties and including gravity effects. It is found that the relative error on the Nusselt number did not exceed 5.5\%. Thus we neglect the buoyancy effects in all the simulations. Therefore, the effect of the temperature variation on the density of the fluid and thus its incompressibility constraints are considered to be unknown.

\section{Results and discussions}

The major purpose of the present study is to represent the effect of the roll-angle on the heat transfer performance. To do so, after validating the obtained results, the flow topology and the temperature distribution are studied along the duct. In addition, investigations are done on the local and global level parameters to check the effect of the roll-angle on the enhancement of the heat transfer.

\subsection{Simulation validation}

Since the velocity and the temperature are set to be uniformed at the inlet of the computational domain, it is considered that the flow is a developing flow. For thermally and hydraulically developing laminar air flow, the results are validated for global Nusselt number using Stephan correlation [21]. The local Nusselt number is represented as followed:

$N u_{x}=7.55+\frac{0.024 x_{*}^{-1.14}\left(0.0179 \operatorname{Pr}^{0.17} x_{*}^{-0.64}-0.14\right)}{\left(1+0.0358 \operatorname{Pr}^{0.17} x_{*}^{-0.64}\right)^{2}}$

with

$x_{*}=\frac{x}{D_{h} \operatorname{RePr}}$

This correlation is valid in the range $0.1 \leq \operatorname{Pr} \leq 1000$ for parallel plate channels in case of uniform wall temperature. Based on equations (12) and (13) the local Nusselt number is calculated and compared with computational results obtained for the empty channel case. Fig. 5 represents the comparison between the correlation (12) and the simulation results: the maximum percentage error is lower than $8 \%$ and the average error is $3.1 \%$ all along the duct, which is a very accurate result compared to commonly encountered values.

\subsection{Flow structure and temperature distribution}

In order to understand the behavior of the flow, the flow structure is first studied for two values of the roll-angle: $\beta=20^{\circ}$ and $\beta=90^{\circ}$, and both Reynolds numbers, i.e. $R e=456$ and 911 .

Fig. 6 represents the helicity distribution along the duct for different cross sections located downstream the VG for the computational domain, for $\beta=20^{\circ}$ and $\beta=90^{\circ}$ and both Reynolds numbers values. Where the helicity is defined as followed: $\lambda=\frac{\vec{v} \cdot \vec{\omega}}{|\vec{v} \cdot \vec{\omega}|}$

where $\vec{v}$ is the spin vector and the $\vec{\omega}$ is the momentum vector.

At $Z=1.3 \mathrm{H}$ the location is just before the leading edge of the VG, whereas at $Z=3.8 H$ the plan is located just after the VG. As shown in Fig. 6, rotating vortices are generated downstream from the VG. For the same Reynolds number and comparing the two cases $\beta=20^{\circ}$ and $\beta=90^{\circ}$, it is clear that how the roll-angle $\beta$ effects on the generation of the vortices and their effect on the flow topology along the duct. For both Reynolds numbers, at $Z=3.8 H$ the helicity values drop to negative, this is due to the reverse vortices generated by the VG. As the roll angle $\beta$ increases, it is clear that the effect of the vortices generated will last longer along the duct until $Z=11.6 \mathrm{H}$ which represents almost the outlet of the duct.

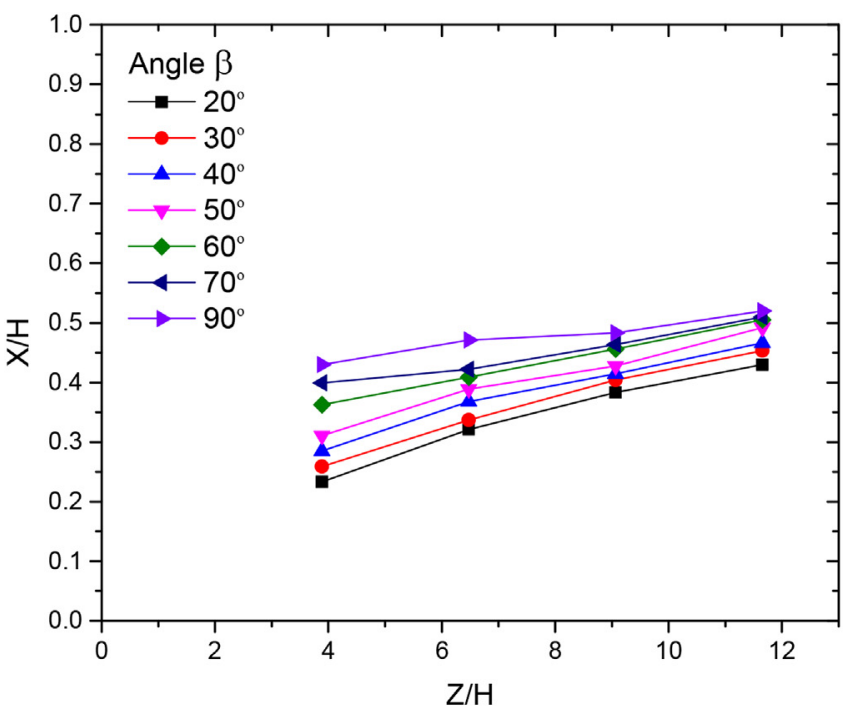

(a)

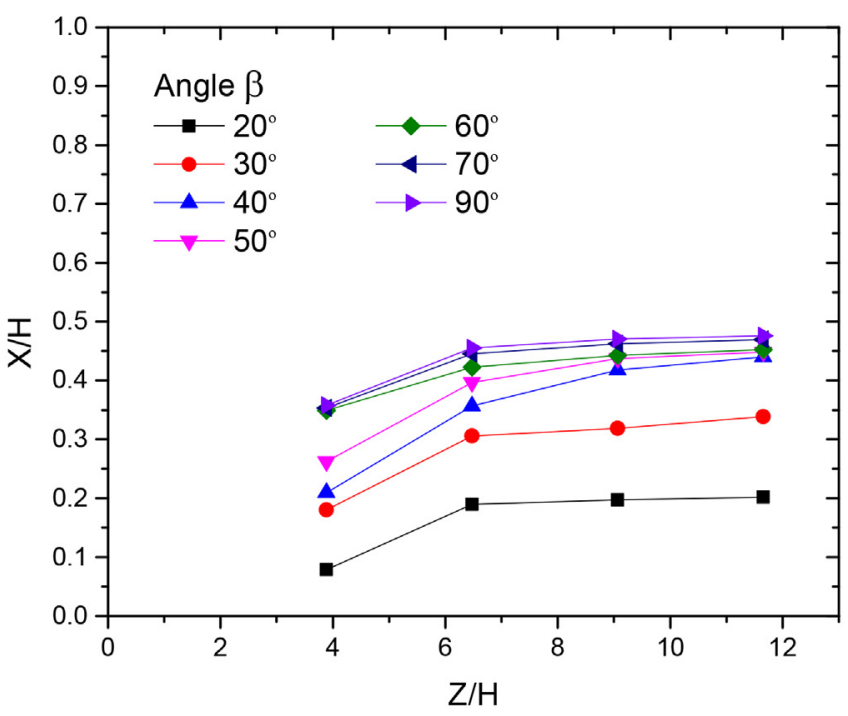

(b)

Fig. 9. Dimensionless location of the main vortex along the duct for (a) $R e=456$ and (b) $R e=911$ 
In Fig. 7 the temperature distribution is represented for both Reynolds number values and roll-angles. From this figure, it is clearly shown that the streamwise vortices previously mentioned affect the temperature distribution downstream the VG, especially for the highest angle. Indeed in the common flow down region, between the two main vortices (i.e. in the symmetry plane of the RWP), hot fluid particles are ejected from the top wall towards the flow core due to upwash effect, while the thickness of the thermal boundary layer on the bottom wall is found to decrease due to downwash effect. In the common flow up region (i.e. between two neighboring vortex pairs) the upwash effect ejects near-wall hot fluid from the bottom wall towards the flow core. This mixing process and thermal boundary thinning are clearly seen for the highest roll-angle value, due to the fact that the vortices are more energetic and cover larger area in the flow cross section. So the heat transfer enhancement seems to be dependent of the roll angle value. This will be assessed and discussed in the next paragraphs by representing the Nusselt number distribution and the vorticity strength streamwise development.

The streamwise variation of the area-weighted average of the helicity is represented in Fig. 8 for all the cases. The locations of the leading and trailing edges of the VG are represented by vertical dashed lines for each case. It is observed that the helicity values start to decrease in the beginning of the curve reaching even negative values at a location just before the VG. After the flow encounters the VG, the averaged helicity is found to increase and reach its maximum value in the very near vicinity of the VG trailing edge because of the generated vortices, for $Z$ between $1.5 \mathrm{H}$ and $3 \mathrm{H}$. It can be noticed that the helicity peak for $\beta=90^{\circ}$ is about 12 times higher than that for $\beta=20^{\circ}$ meaning higher energetic vortices.

For $20^{\circ}<\beta<60^{\circ}$ the profile of the vorticity streamwise evolution is similar having a maximum at the tail of VG and decreasing continuously along the duct because of the dissipation of the LV. Whereas for higher $\beta$ values $\left(70^{\circ}\right.$ and $90^{\circ}$ ), secondary peaks are generated along with the duct due to the secondary vortices generated.

For $R e=911$, the value of the helicity peak increases by a factor of 3 compared to that of $R e=456$. As a profile of the curve, for all the angles the maximum value of the helicity is at the tail of the VG. For small angle values $20^{\circ}<\beta<40^{\circ}$, after reaching to the maximum value it fades away along the duct due to the dissipation of the LV. For $50^{\circ}<\beta<90^{\circ}$ a secondary peak is generated each at a different location along the duct. It is interesting to notice that for $\beta=60^{\circ}$, $\beta=70^{\circ}$ and $\beta=90^{\circ}$, similar values of helicity are obtained near VG whereas along the downstream direction of the duct, both $\beta=60^{\circ}$ and $\beta=70^{\circ}$ have a secondary peak due to the secondary vortices. The secondary peak values of both $\beta=60^{\circ}$ and $\beta=70^{\circ}$ are almost equal, but the primary peak value of the $\beta=70^{\circ}$ is higher than that of the $\beta=60^{\circ}$. On the other hand, for $\beta=90^{\circ}$, the primary peak value is higher than that of all the cases but the effect of the secondary vortices are smaller compared to that of $\beta=60^{\circ}$ and $\beta=70^{\circ}$. It can be concluded that based on the helicity values, $\beta=70^{\circ}$ appear to be the optimal angle that can be considered for $R e=911$ compared to the other values of roll-angle.

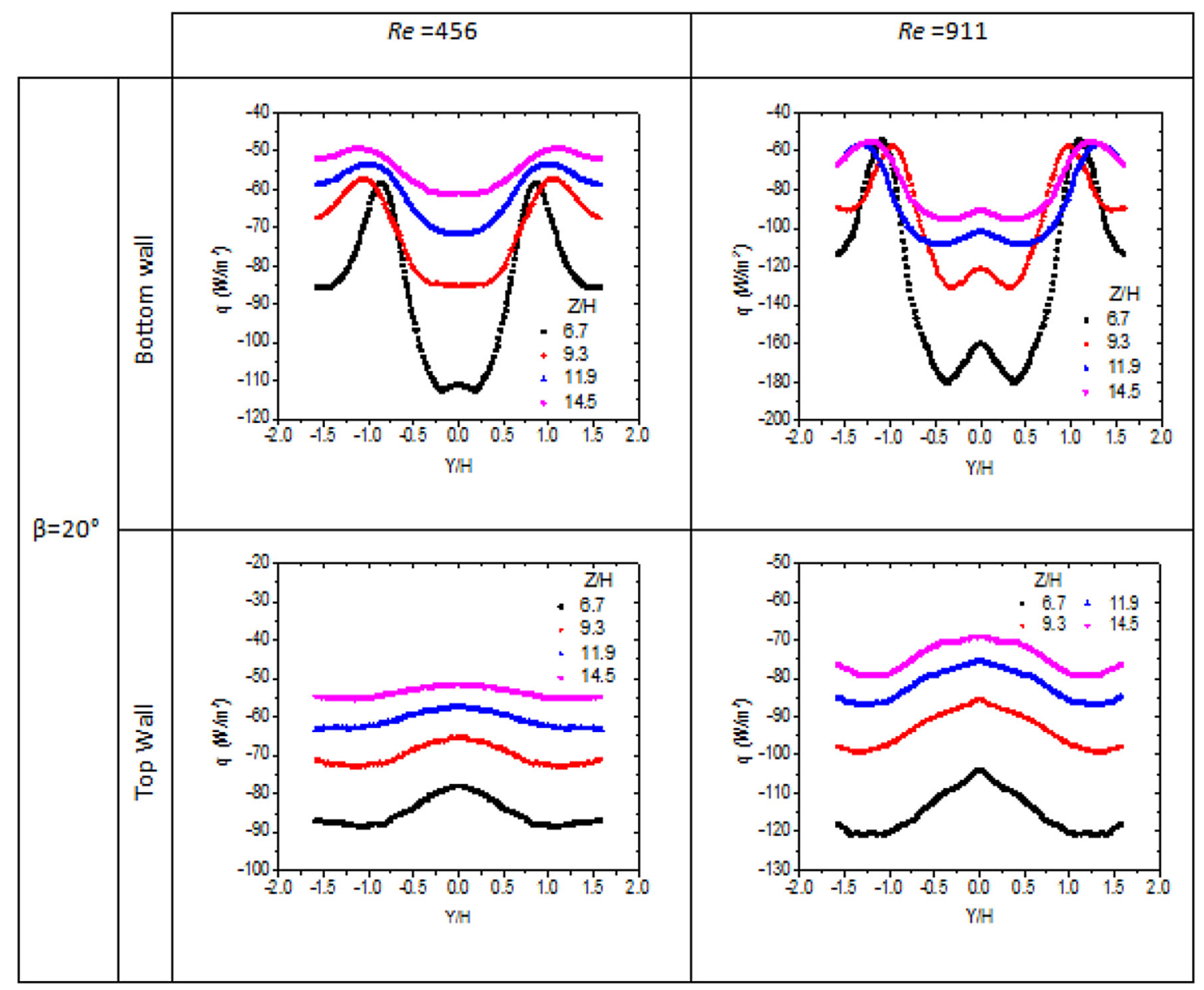

Fig. 10. Heat flux both on bottom and top walls for different locations away from the VG for $\operatorname{Re}=456$ and $\operatorname{Re}=911, \beta=20^{\circ}$. 
In Fig. 9 the streamwise variation of the dimensionless location of the main vortex is presented, where the distance between the vortex core and the bottom wall is measured. In Fig. 9 it is observed that for $R e=456$, the position of the core of the main vortex starts in an increasing profile for all the values of roll-angle just after the tail of the VG. It is observed that by increasing the value of $\beta$, the position of the main vortex increases, reaching a maximum location at a value of $X=0.5 H$ for $\beta=90^{\circ}$, which represents the half of the duct height.

For the case of $R e=911$ the position of the core of the main vortex increases with the increase of the $\beta$. By comparing both Reynolds number, it is obvious that for $R e=911$ the increase is more rapid, where for high values of $\beta$, the location of the core of the main vortex reaches to the half of the duct height more quickly. On the other hand for low values of $\beta$, the location of the core of the main vortex will not reach to that height.

As the dimensionless location of the core of the main vortex reaches to the half of the duct height, it leads to a better mixing of the fluid in the duct. As a result, the heat transfer rate increases, and then the Nusselt number is affected. The variation in the Nusselt number is studied in the coming sections, in order to provide a clear view of the effect of the roll-angle $\beta$ on the heat transfer enhancement.

\subsection{Local performance}

In order to study the local performance, different locations behind the VG are considered on both bottom and top walls where the wall heat flux density is studied for each case. Fig. 10 and Fig. 11 represent the results obtained respectively for $\beta=20^{\circ}$ and $\beta=90^{\circ}$ for both Reynolds numbers. At a short distance from the VG (Z/ $H=6.7$ ) the secondary vortex can still be seen. Along the downstream, the heat flux density is dominated by the main vortices. By increasing the distance $Z / H$, the heat flux density decreases and the vortices diverge.

By comparing Figs. 10 and 11, the effect of the roll-angle $\beta$ is introduced on the local performance. Whereas for $\beta=90^{\circ}$, both bottom and top wall heat flux values are higher than that of $\beta=20^{\circ}$.

The streamwise variation of the cross-section averaged Nusselt number is presented in Fig. 12 and Fig. 13 for bottom and top walls respectively, on which the vertical dashed lines represent the leading and trailing edges of the VG. The average Nusselt number on each cross section is obtained by the following equation:

$$
\begin{aligned}
& N u=\frac{2 H}{k_{f}} \frac{q^{\prime \prime}}{\left(T_{w}-T_{f}\right)} \\
& N u(z)=\frac{2 H}{k_{f}} \frac{q^{\prime \prime}(z)}{\left(T_{w}-T_{f}(z)\right)}
\end{aligned}
$$

where $q$ " is the average wall heat flux density on both walls, $q$ " $(Z)$ is the average wall heat flux density at a given $(Z)$ streamwise location, $k_{f}$ is the thermal conductivity of the fluid, $T_{w}$ the wall surface temperature and $T_{f}(Z)$ the fluid bulk temperature at a given $Z$ crosssection location.

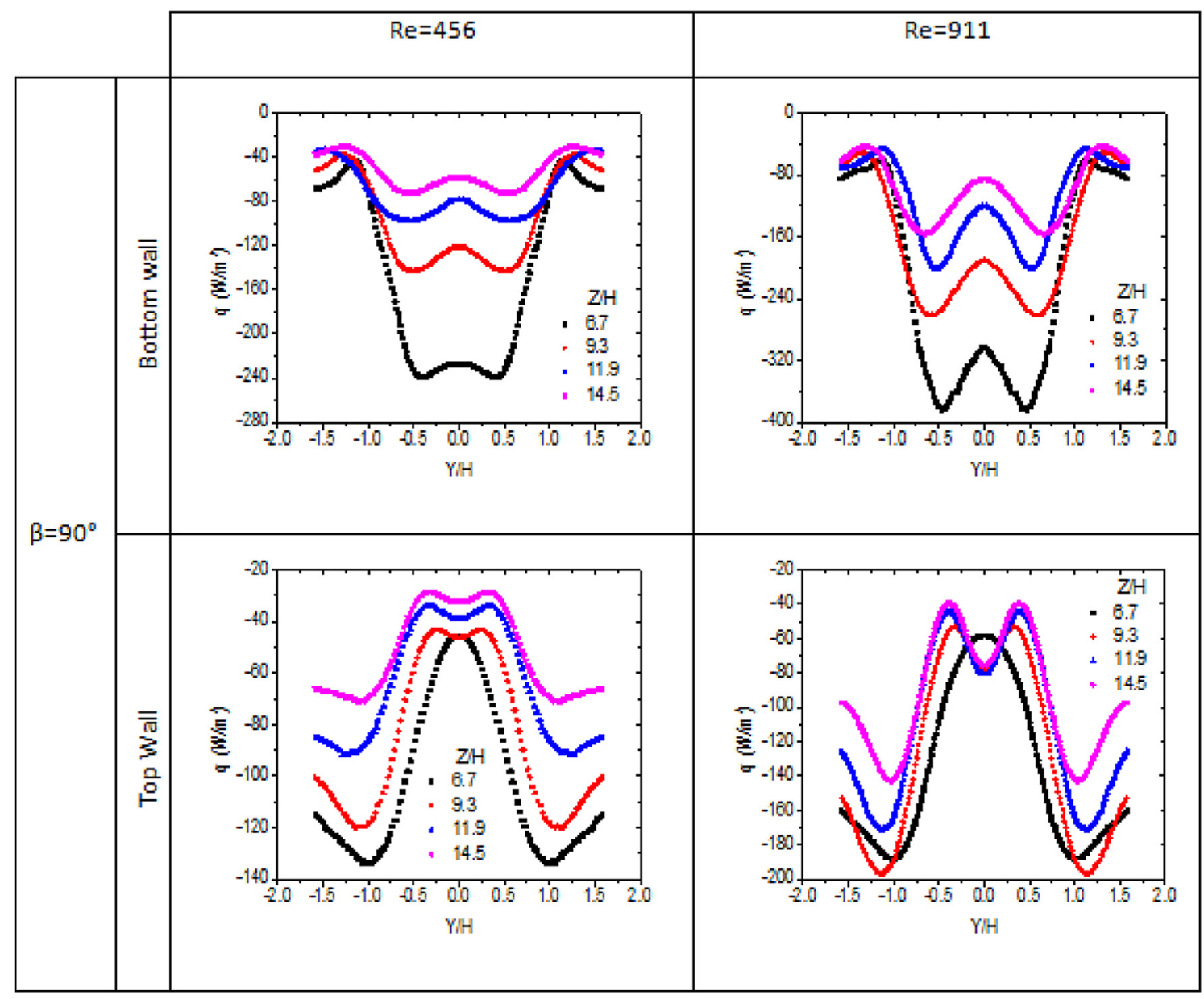

Fig. 11. Heat flux both on bottom and top walls for different locations away from the VG for $R e=456$ and $R e=911, \beta=90^{\circ}$. 


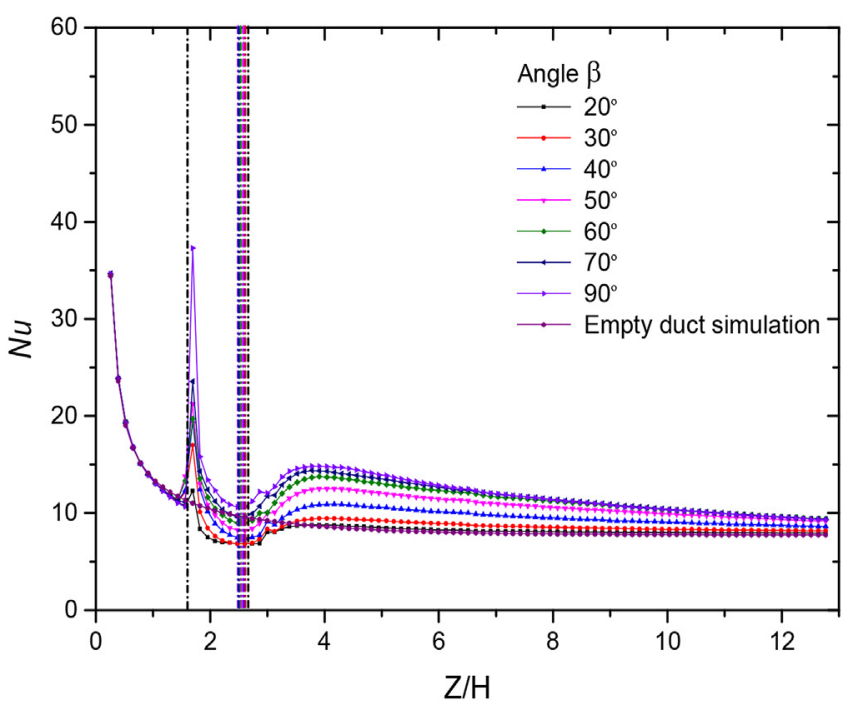

(a)

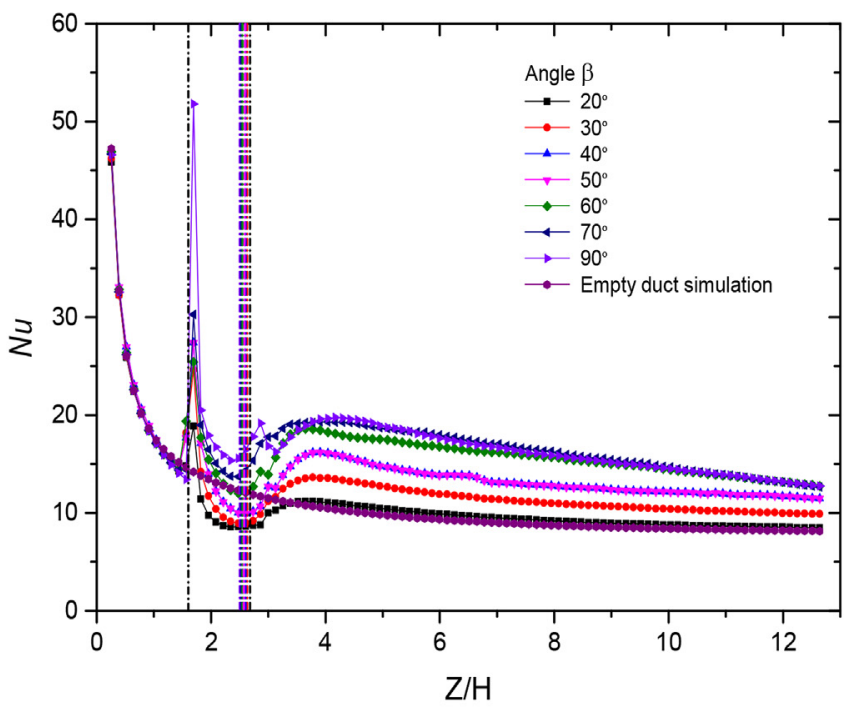

(b)

Fig. 12. Bottom wall and VG Nusselt number for (a) $R e=456$ and (b) $R e=911$.

The streamwise distribution of the span-averaged Nusselt number is plotted for all values of roll-angles $\beta$ as well as for the case of the empty duct. For both Reynolds numbers, similar profile is obtained, where at the inlet of the duct, the Nusselt number values decrease until the location of $Z=1.6 \mathrm{H}$ which represents the head of the VG. At that location the Nusselt number value starts to increase due to the heat transfer enhancement until it reaches the tail of the VG where it drops to the minimum. For low values of $\beta$ the Nusselt number value drop is found to be even below that observed for the empty duct channel. This drop is seen at a location of $Z=2.3 \mathrm{H}$ which is at the tail of the VG. After the drop a second peak is generated where the value of the Nusselt number increases at a location of just after the VG $(Z=2.5 H)$. For all the values of angle $\beta$, a similar profile is obtained where a second peak is generated just after the VG after which it decreases with a small oscillation along the longitudinal direction of the duct.

The drop in the Nusselt number curve below the value observed for the empty duct channel occurs only on the bottom wall. In Fig. 13, it is clear that the Nusselt number for the top wall decreases

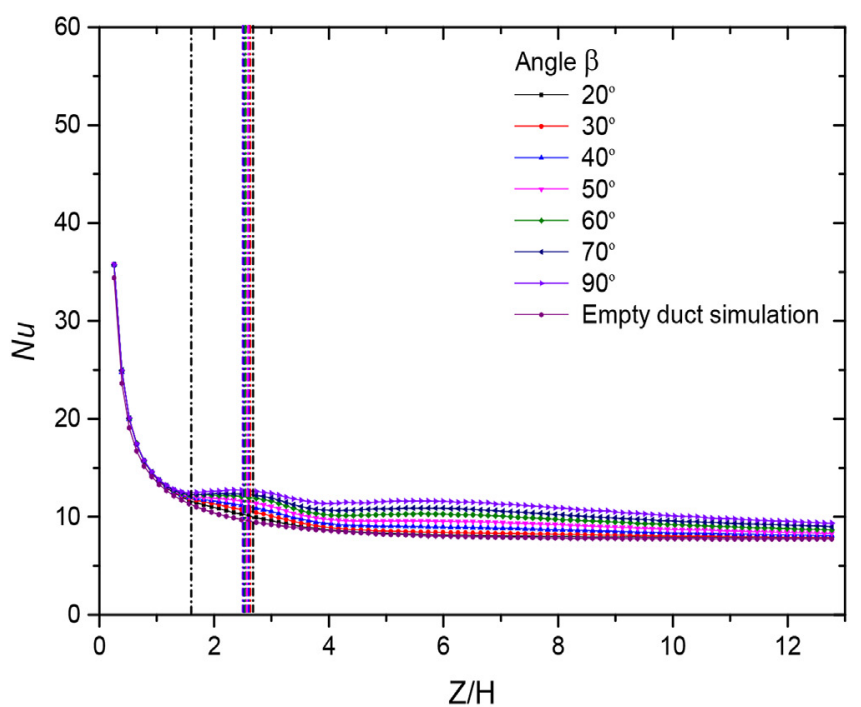

(a)

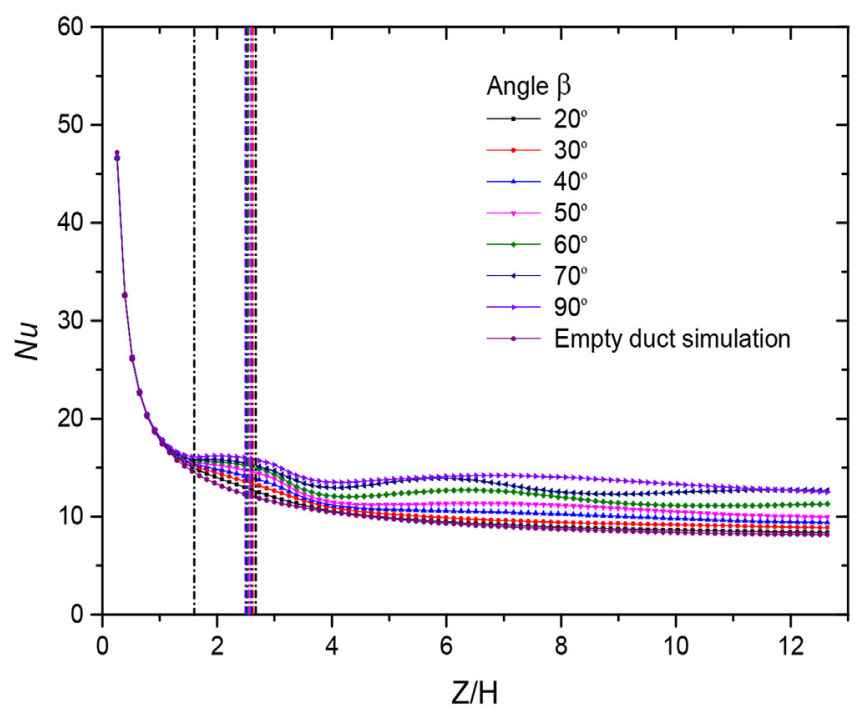

(b)

Fig. 13. Top wall Nusselt number for (a) $R e=456$ and (b) $R e=911$.

in the inlet of the duct, until a location of $Z=1.6 \mathrm{H}$, then the value starts to increase reaching their maximum at $Z=2.3 H$ which represents the tail of the VG. After which the curves start to drop along the longitudinal direction of the duct with.

To understand the drop of the Nusselt number below that of the empty duct at the VG location in some studied cases, a study is performed to check the interaction between the heat flux and the near-wall velocity field on the bottom wall and the top wall. In Fig. 14 and Fig. 15, the boundary heat flux and the velocity field are displayed for roll-angles equal to $20^{\circ}$ and $90^{\circ}$ and Reynolds numbers values respectively 456 and 911, the planes are taken at a distance of $X=H / 8$ away from the bottom or top wall, knowing that $X=0$ represents the bottom wall location. It appears clearly that the velocity value for the bottom wall case at a location under the area of the VG is nearly zero. For low values of $\beta$ this area of velocity null is greater than for the case of high $\beta$, implying that there is a bigger region where a bigger dead zone is created under the VG, where due to zero velocity there is almost no heat transfer due to convection. As a result, the Nusselt number value is dropping even 


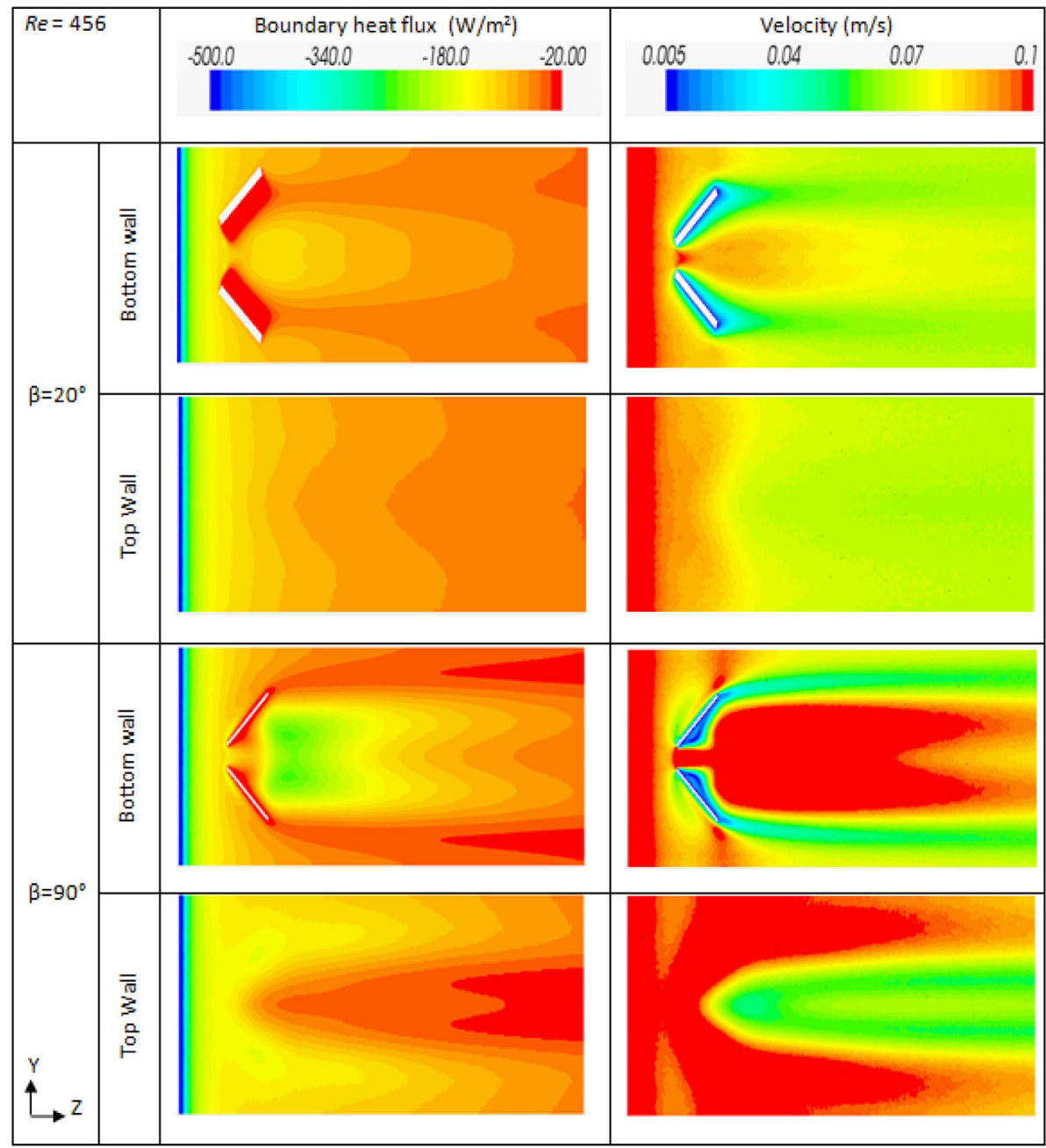

Fig. 14. Heat flux and velocity fields at location $X=H / 8$ away from either bottom or top walls, $R e=456$.

below that of the empty duct channel case. On the other hand, since there is no VG placed on the top wall, there is no dead zone created there. Thus, there is convective heat transfer on the top wall enhanced by the presence of the VG on the bottom wall. This is supported by the values of the Nusselt number, represented in Fig. 13 for the top wall, on which for all the values of roll-angle $\beta$, the curves are above the values of the empty duct channel.

Fanning friction factor $f$ is calculated from the pressure gradient obtained from the simulation. As Darcy's friction can be calculated by:

$f_{\text {darcy }}=2 \frac{D_{h}}{L} \cdot \frac{\Delta p}{\rho U^{2}}=\frac{4 H \Delta p}{L \rho U^{2}}$

thus

$f=\frac{f_{\text {darcy }}}{4}=\frac{H \Delta p}{L \rho U^{2}}$

where $H$ is the height of the duct $(\mathrm{m}), \Delta p$ is the pressure drop (Pa) between the inlet and the outlet, $\mathrm{L}$ is the length of the duct $(\mathrm{m}), \rho$ is the fluid density $\left(\mathrm{kg} / \mathrm{m}^{3}\right)$ and $U$ is the mean flow velocity $(\mathrm{m} / \mathrm{s})$.

In order to calculate the local Fanning friction factor $f(Z)$, the following relation is used:
$f(Z)=\frac{f(Z)_{\text {darcy }}}{4}=\frac{H \Delta p(Z)}{Z \rho U^{2}}$

where $\Delta p(Z)$ is the pressure drop (Pa) between the inlet and the cross section at location $Z$.

In Fig. 16, the streamwise evolution of the friction factor is represented. For both Reynolds numbers the profile of the curve for high roll-angle values $\left(\beta=50^{\circ}, \beta=60^{\circ}, \beta=70^{\circ}\right.$ and $\left.\beta=90^{\circ}\right)$ is similar where it starts to drop along the length of the duct until it reaches the leading edge of the VG. At that point a gradual increase starts to appear reaching its maximum value at the tail of the VG, after which the curve continues to drop making an asymptote with the empty channel curve. On the other hand, for low values of rollangle $\left(\beta=20^{\circ}, \beta=30^{\circ}\right.$ and $\left.\beta=40^{\circ}\right)$ the profile starts to drop along the length of the duct until it reaches the leading edge of the VG. At that point an increase starts to appear that last for small values of $Z$ / $H$, after which the curve continues to drop making an asymptote with the empty channel curve. For the case $R e=456$ the maximum peak obtained is higher than that of the case $R e=911$, knowing that the friction factor is inversely proportional to the fluid velocity which is by itself directly proportional to the Reynolds number. On the other hand the pressure drop $\Delta p$ for $R e=911$ is higher than that for $R e=456$, but the ratio of it with respect to the velocity square is lower than that for $R e=456$ 


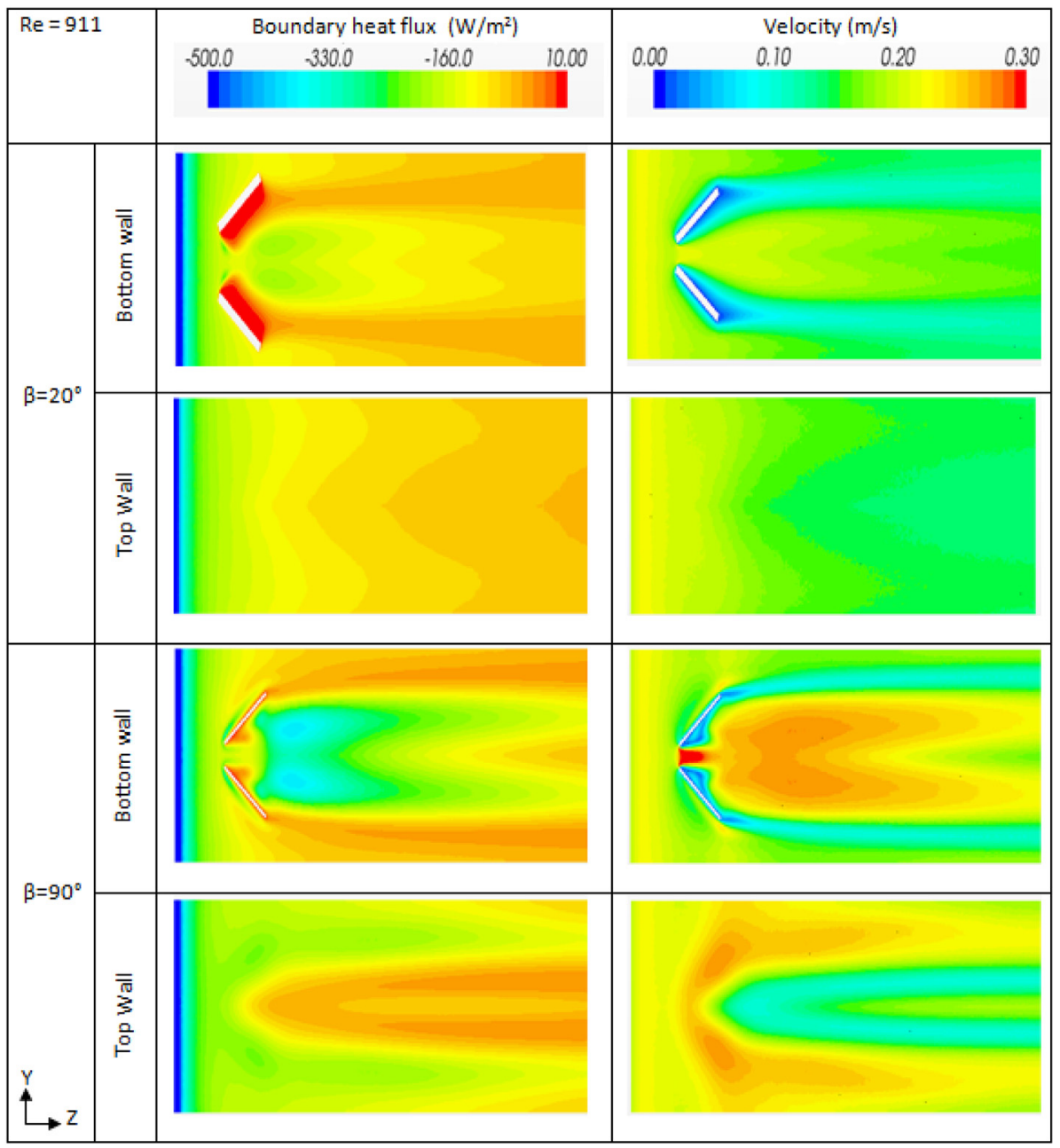

Fig. 15. Heat flux and velocity fields at location $X=H / 8$ away from either bottom or top walls, $R e=911$.

\subsection{Global performance}

In order to study the global effect of the transverse angle $\beta$ of the VG on the enhancement of the heat transfer, global values of the Nusselt number, friction factor and the enhancement factor are presented and discussed in this section.

Fig. 17 represents the Nusselt number plotted for both Reynolds numbers, versus the roll-angle $\beta$. The Nusselt number is calculated using equation (4). For both values of Reynolds numbers, the Nusselt number is found to monotonically increase with the value of the roll-angle. $\beta=0$ represents the empty duct channel. By comparing the empty duct values of Nusselt number with the other cases, for all the case the $N u$ value increases. A similar profile is obtained for both Reynolds values.

The global friction factor is also plotted for both Reynolds numbers versus the roll-angle in Fig. 18. The friction factor is calculated using equation (13). As the roll-angle $\beta$ increases, the friction factor increases too. For high values of angle $\beta$ the pressure drop is high. As a result of this, the pressure gradient is increased leading to a high value of friction factor. $\beta=0$ represents the empty duct channel. By comparing the empty duct values of friction factor with the other cases, for all the case the friction factor value increases. A similar profile is obtained for both Reynolds values.

Based on the previously mentioned relations of the Nusselt number and the friction factor, an enhancement factor is calculated using the following relation:

$$
\left.\eta=\left(\frac{N u}{N u_{0}}\right)\left(\frac{f}{f_{0}}\right)^{(-1 / 3}\right)
$$

where $N u_{0}$ and $f_{0}$ are the values of Nusselt number and friction factor respectively for the empty duct channel.

Fig. 19 represents the enhancement factor for both Reynolds numbers. For $R e=456$, the value of the enhancement factor starts to increase monotonically with the increase of the transverse angle $\beta$ reaching its maximum value of 1.2 at $\beta=90^{\circ}$. On the other hand for the case of $R e=911$ the profile of the enhancement factor curve starts to increase with the increase of the roll-angle $\beta$ until it reaches a maximum value of 1.32 at $\beta=70^{\circ}$, after which its value slightly drops to a value of 1.3 at $\beta=90^{\circ}$. Thus, it can be considered that for the case $R e=456$ the optimum value of $\beta$ is the highest angle $90^{\circ}$. Nevertheless, for the case $R e=911$ the optimum rollangle among those tested values of $\beta$ to provide the best enhancement is found to be $70^{\circ}$ and not the highest angle. This is also supported by the helicity curve shown in Fig. 6 where the secondary vortices are detected.

By comparing the values of the enhancement factor of both Reynolds numbers, for all the values of $\beta$, that of $R e=911$ have a higher percentage of enhancement than that of $R e=456$. For $\beta=70^{\circ}$, the enhancement factor percentage of $R e=456$ is $1.18 \%$, whereas for $R e=911$ is $1.32 \%$ having a difference of $0.14 \%$. On the 


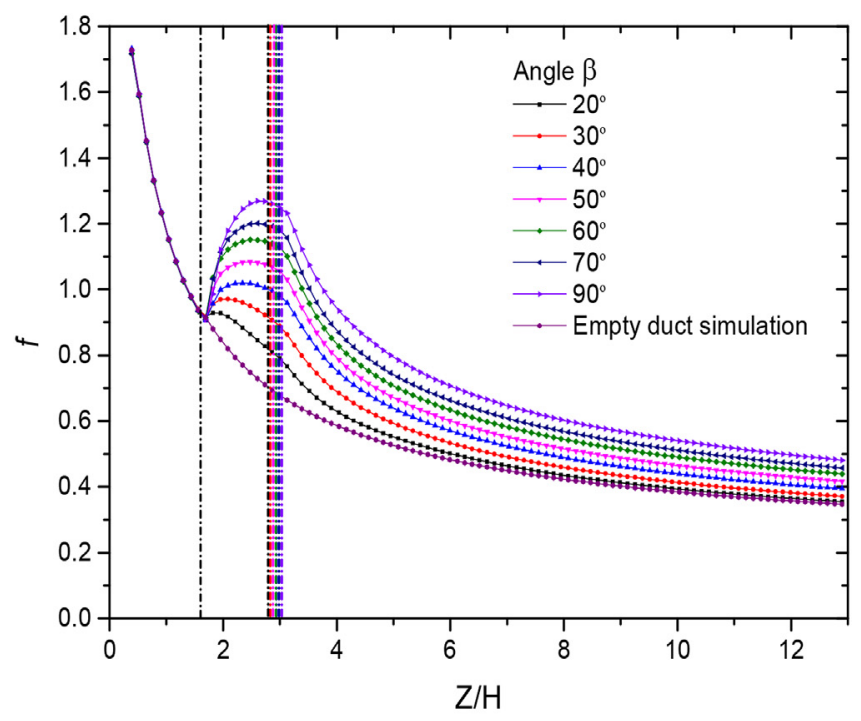

(a)

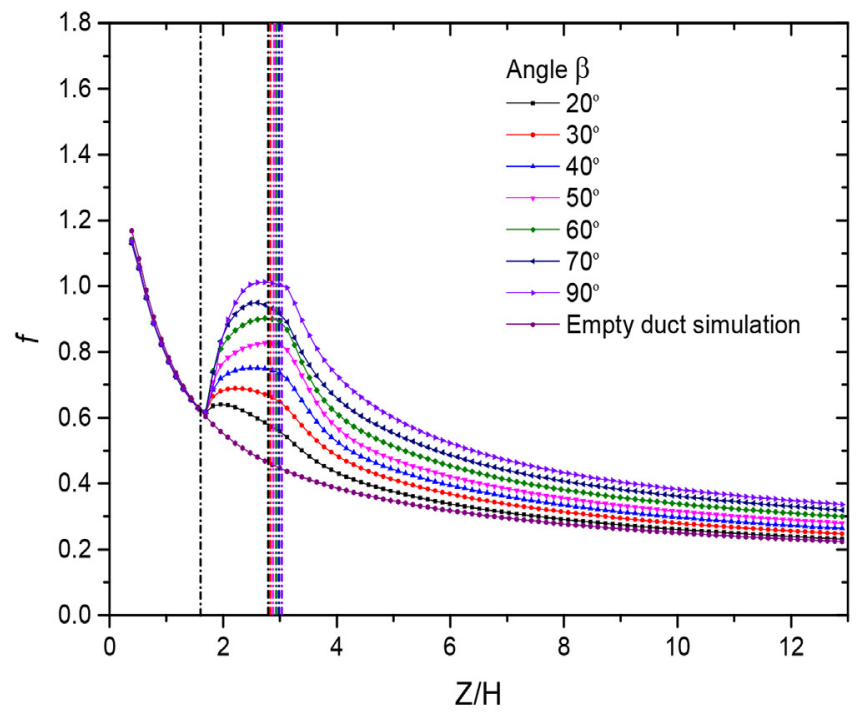

(b)

Fig. 16. Friction factor streamwise evolution for (a) $R e=456$ and (b) $R e=911$.

other hand, for $\beta=20^{\circ}$, the enhancement factor percentage of $R e=456$ is $1.02 \%$, whereas for $R e=911$ is $1.027 \%$ having a difference of $0.007 \%$.

\section{Conclusion}

In this study, heat transfer and fluid flow characteristics in a rectangular duct with RWPVG inserted on its bottom wall are numerically investigated. For different values of generator's rollangle $(\beta)$ both local and global hydrodynamic and thermal parameters are calculated and studied. The goal of these studies is to figure out the effect of the roll-angle on the heat transfer enhancement. The main outcomes of this study can be summarized as followed.

For high values of roll-angle (close to $\beta=90^{\circ}$ ), it is observed that the helicity increases just after the flow encounters the VG where the vortices are first formed. The helicity peak for $\beta=90^{\circ}$ is about 12 folds higher than that for $\beta=20^{\circ}$ meaning more energetic vortices. Moreover, increasing the mean flow velocity, i.e. the

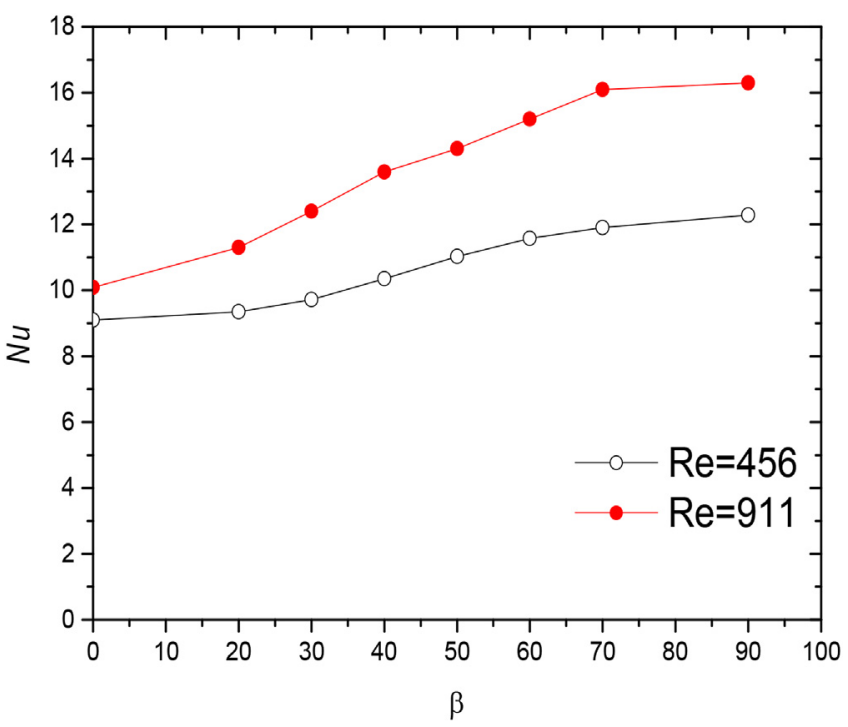

Fig. 17. Global Nusselt number for $R e=456$ and $R e=911$.

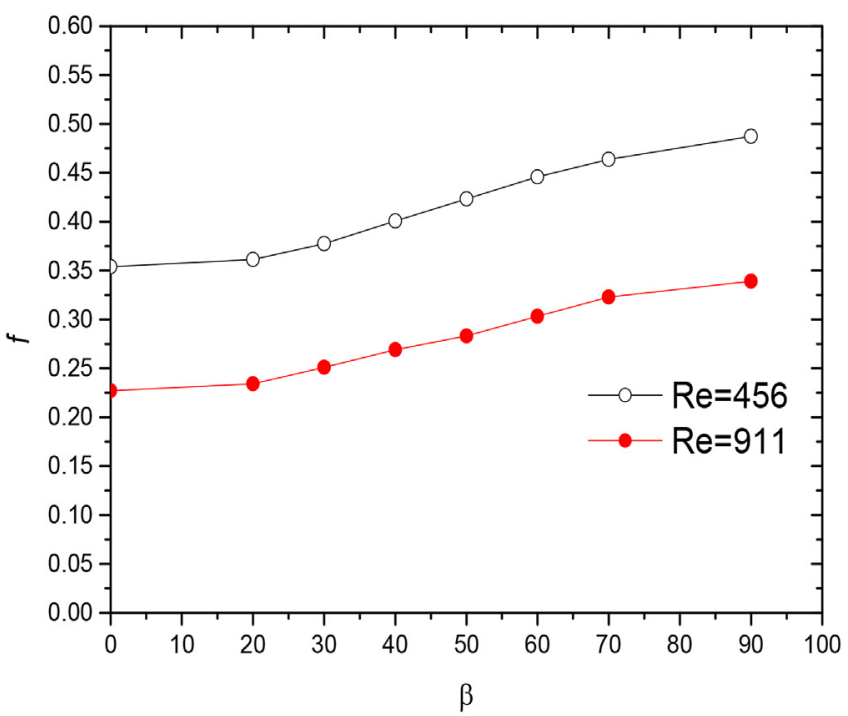

Fig. 18. Global friction factor for $R e=456$ and $R e=911$.

Reynolds number, leads to increase the helicity. It is observed that for $R e=456$, the dimensionless $X / H$ position of the main vortex measured from the bottom wall, starts at low position and increases along the duct until it reaches a maximum value of around 0.5 representing the middle of the duct for the highest value of rollangle $\beta$. Whereas for the case of higher Reynolds number, dimensionless location of the main vortex is increased suddenly at the $Z$ / $H=6.5$ and after that in continuous to increase gradually along the length of the duct. For $R e=911$, the highest value of $X / H=0.5$ is reached by the high values of $\beta$. Whereas for $\beta=30^{\circ}$ the value of the $X / H=0.35$ and for $\beta=20^{\circ}, X / H=0.2$.

It is crucial to take into consideration that by increasing the rollangle value, not only the heat transfer will be enhanced but on the other hand the pressure drop will increase too. In order to be able to make decision for this challenging issue the enhancement factor is studied where both the heat transfer and the pressure drop are taken into consideration.

For $R e=456$, it is shown that the enhancement factor monotonically increases with the increase of the roll-angle $\beta$, reaching its 


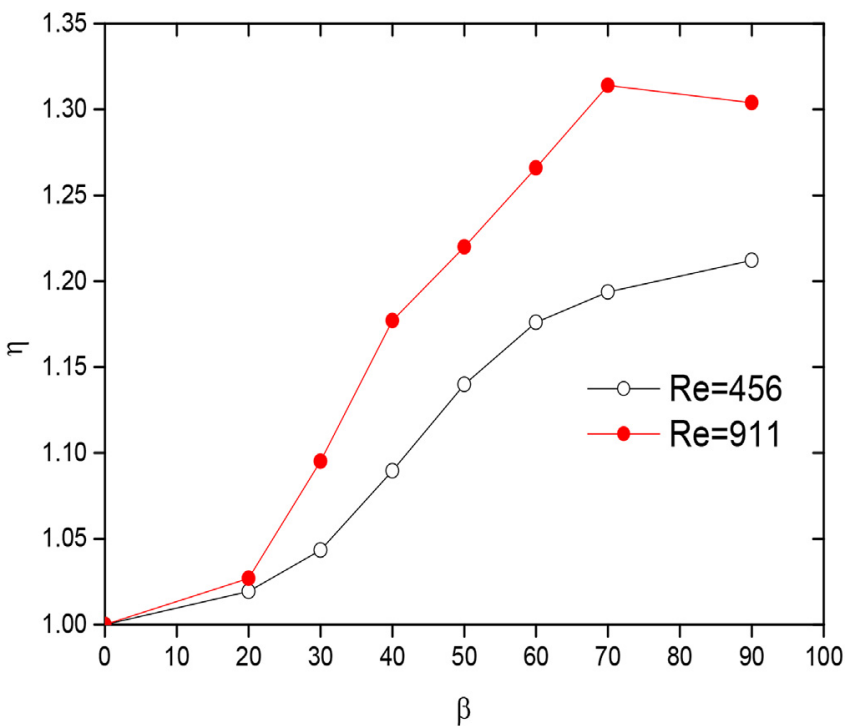

Fig. 19. Enhancement factor for $R e=456$ and $R e=911$.

maximum value of 1.2 at $\beta=90^{\circ}$. On the other hand for the case of $R e=911$ the profile of the enhancement factor starts to increase with the roll-angle $\beta$ until it reaches a maximum value of 1.32 near $\beta=70^{\circ}$. Thus, it can be considered that for $R e=911$ the optimum roll-angle among those tested values of $\beta$ which leads to the best enhancement is $70^{\circ}$ and not the highest angle, while for $R e=456$ the optimum value of $\beta$ among those tested is the highest angle $90^{\circ}$.

After all these investigations, it is crucial to mention that the roll-angle $\beta$ has a significant effect on the enhancement of the heat transfer. It is interesting to study the $70^{\circ}$ case in place of the perpendicular RWPVG for further investigations. As an advancement of the obtained results, few more values of $\beta$ can be added for values near to $70^{\circ}$ in order to find the exact value of the optimum angle. Due to different values of obtained for different Reynolds numbers, it is interesting to go to higher Reynolds and even study in the turbulent regime too.

\section{References}

[1] Khoshvaght-Aliabadi M, Zangouei S, Hormozi F. Performance of a plate-fin heat exchanger with vortex-generator channels: 3D-CFD simulation and experimental validation. Int. J. Therm. Sci. 2015;88:180-92.

[2] Ali S, Habchi C, Menanteau S, Lemenand T, Harion JL. Heat transfer and mixing enhancement by free elastic flaps oscillation. Int. J. Heat Mass Transf. 2015;85: 250-64.

[3] Alahyari Beig S, Mirzakhalili E, Kowsari F. Investigation of optimal position of a vortex generator in a blocked channel for heat transfer enhancement of electronic chips. Int. J. Heat Mass Transf. 2011;54:4317-24.

[4] Jiang Y, Zheng Q, Dong P, Zhang H, Yu F. Research on heavy-duty gas turbine vane high efficiency cooling performance considering coolant phase transfer. Appl. Therm. Eng. 2014;73:1175-91.

[5] Basu DN, Bhattacharyya S, Das PK. A review of modern advances in analyses and applications of single-phase natural circulation loop in nuclear thermal hydraulics. Nucl. Eng. Des. 2014;280:326-48.

[6] Fiebig M. Vortices Generators and Heat Transfer. Chem. Eng. Res. Des. 1998;76:108-23.

[7] Habchi C, Russeil S, Bougeard D, Harion JL, Lemenand T, Della Valle D, et al. Enhancing heat transfer in vortex generator-type multifunctional heat exchangers. Appl. Therm. Eng. 2012:38:14-25.

[8] Anxionnaz Z, Cabassud M, Gourdon C, Tochon P. Heat exchanger/reactors (HEX reactors): concepts, technologies: state-of-the-art. Chem. Eng. Process. Process Intensif. 2008;47:2029-50.

[9] Ghanem A, Habchi C, Lemenand T, Della Valle D, Peerhossaini H. Energy efficiency in process industry - high-efficiency vortex (HEV) multifunctional heat exchanger. Renew. Energy 2013;56:96-104.

[10] Tiggelbeck S, Mitra NK, Fiebig M. Flow Structure and Heat Transfer in a channel with multiple longitudinal vortex generators. Exp. Therm. Fluid Sci. 1992;5:425-36.

[11] Jacobi AM, Shah RK. Heat transfer surface enhancement through the use of longitudinal vortices: a review of recent progress experimental thermal and fluid science, vol. 11; 1995. p. 295-309.

[12] Habchi C, Lemenand T, Della Valle D, Pacheco L, Le Corre O, Peerhossaini H. Entropy production and field synergy principle in turbulent vortical flows. Int. J. Therm. Sci. 2011;50:2365-76.

[13] Mohand Kaci H, Habchi C, Lemenand T, Della Valle D, Peerhossaini H. Flow structure and heat transfer induced by embedded vorticity. Int. J. Heat Mass Transf. 2010;53:3575-84.

[14] Zhou G, Feng Z. Experimental investigations of heat transfer enhancement by plane and curved winglet type vortex generators with punched holes. Int. J. Therm. Sci. 2014;78:26-35.

[15] Wang Chi-Chuan, Chen Kuan-Yu, Lin Yur-Tsai. Investigation of the semidimple vortex generator applicable to fin-and-tube heat exchangers. Appl. Therm. Eng. 5 September 2015;88:192-7.

[16] Wu JM, Tao WQ. Numerical study on laminar convection heat transfer in a channel with longitudinal vortex generator. Part B: parametric study of major influence factors. Int. J. Heat Mass Transf. 2008;51:3683-92.

[17] Wu JM, Tao WQ. Numerical study on laminar convection heat transfer in a rectangular channel with longitudinal vortex generator. Part A: verification of field synergy principle. Int. J. Heat Mass Transf. 2008;51:1179-91.

[18] Tiggelbeck S, Mitra NKFM. Comparison of wing-type vortex generators for heat transfer enhancement in channel flows. J. Heat Transf. 1994;116:880-5.

[19] CD-Adapco, Star-CCM+, in: user guide version 8.06. 2013.

[20] Celik IB, Ghia U, Roache PJ, Freitas CJ, Coleman H, Raad PE. Procedure for estimation and reporting of uncertainty due to discretization in CFD applications. J. Fluid. Eng. 2008;130:078001. 078001.

[21] Lu G, Zhou G. Numerical simulation on performances of plane and curved winglet type vortex generator pairs with punched holes. Int. J. Heat Mass Transf. 2016;102:679-90.

[22] Dezan DJ, Salviano LO, Yanagihara JI. Heat transfer enhancement and optimization of flat-tube multilouvered fin compact heat exchangers with deltawinglet vortex generators. Appl. Therm. Eng. 2016;101:576-91. 